\title{
Effective Transactional Memory Execution Management for Improved Concurrency
}

\author{
M. A. GONZALEZ-MESA, ELADIO GUTIERREZ, EMILIO L. ZAPATA, \\ and OSCAR PLATA, Dept. of Computer Architecture, University of Málaga
}

This article describes a transactional memory execution model intended to exploit maximum parallelism from sequential and multithreaded programs. A program code section is partitioned into chunks that will be mapped onto threads and executed transactionally. These transactions run concurrently and out of order, trying to exploit maximum parallelism but managed by a specific fully distributed commit control to meet data dependencies. To accomplish correct parallel execution, a partial precedence order relation is derived from the program code section and/or defined by the programmer. When a conflict between chunks is eagerly detected, the precedence order relation is used to determine the best policy to solve the conflict that preserves the precedence order while maximizing concurrency. The model defines a new transactional state called executed but not committed. This state allows exploiting concurrency on two levels: intrathread and interthread. Intrathread concurrency is improved by having pending uncommitted transactions while executing a new one in the same thread. The new state improves interthread concurrency because it permits out-of-order transaction commits regarding the precedence order. Our model has been implemented in a lightweight software transactional memory system, TinySTM, and has been evaluated on a set of benchmarks obtaining an important performance improvement over the baseline TM system.

Categories and Subject Descriptors: B.3 [Hardware]: Memory Structures; C.1.4 [Computer Systems Organization]: Processor Architectures-Parallel architectures; D.2.8 [Software Engineering]: MetricsComplexity measures, performance measures

General Terms: Design, Experimentation, Performance

Additional Key Words and Phrases: Transactional memory, concurrency exploitation, dependence analysis, optimistic concurrency, program parallelization

ACM Reference Format:

M. A. Gonzalez-Mesa, Eladio Gutierrez, Emilio L. Zapata, and Oscar Plata. 2014. Effective transactional memory execution management for improved concurrency. ACM Trans. Architec. Code Optim. 11, 3, Article 24 (July 2014), 27 pages.

DOI: http://dx.doi.org/10.1145/2633048

\section{INTRODUCTION}

The current trend in commodity computers is providing users with a large amount of computational power in terms of multiple processor cores sharing memory [Geer 2005]. Current processors integrate in the same chip a number of cores that communicate through a multilevel, partially shared on-chip cache hierarchy. Parallel execution, in

This work has been supported by the government of Spain with projects CICYT TIN2010-16144 and Consolider Ingenio 2010 CSD2007-00050, and the Autonomic Government of Andalucía (JA TIC-4341).

Authors' addresses: M. A. Gonzalez-Mesa, E. Gutierrez, E. L. Zapata, and O. Plata, Dept. of Computer Architecture, University of Málaga, 29071 Málaga, Spain; emails: magonzalez@ac.uma.es, eladio@uma.es, ezapata@ac.uma.es, oplata@uma.es.

Permission to make digital or hard copies of part or all of this work for personal or classroom use is granted without fee provided that copies are not made or distributed for profit or commercial advantage and that copies show this notice on the first page or initial screen of a display along with the full citation. Copyrights for components of this work owned by others than ACM must be honored. Abstracting with credit is permitted. To copy otherwise, to republish, to post on servers, to redistribute to lists, or to use any component of this work in other works requires prior specific permission and/or a fee. Permissions may be requested from Publications Dept., ACM, Inc., 2 Penn Plaza, Suite 701, New York, NY 10121-0701 USA, fax +1 (212) 869-0481, or permissions@acm.org.

(c) 2014 ACM 1544-3566/2014/07-ART24 $\$ 15.00$

DOI: http://dx.doi.org/10.1145/2633048 
the form of multiple concurrent threads sharing memory, must be used by programmers in order to exploit the available hardware and extract maximum performance.

In contrast to ILP (instruction-level parallelism), thread-level parallelism cannot be entirely exploited by hardware; some software (compiler, library, runtime) and/or programmer (language) support is required. One approach is automatic parallelization, where the programmer is not involved [Midkiff 2012]. The compiler, with the help of library or runtime support, is in charge of analyzing dependencies in the program and generating a correct parallel version. It is known that this kind of analysis is very complex and conservative, especially with codes that use pointers and dynamic data structures, so it is only successful for regular applications. In addition, in many cases, codes offer hidden parallelism that a static parallelizer cannot discover until program execution. A solution, with limited success, is to speculate data dependencies at runtime, a technique called TLS (thread-level speculation) or SpMT (speculative multithreading) [Sohi et al. 1995].

Another approach is specifying explicit parallelism by the programmer at the language level. Reasoning about the semantics of the application, the programmer is now in charge of the analysis of the program to define the parallel threads and their synchronization. In general, performance of multithreaded applications is limited by how the parallel threads are synchronized. The traditional approach for synchronizing threads has been to use barriers and locks (mutual exclusion), which are often responsible for degrading parallel performance and are not scalable. Fine-grain locking can improve opportunities for parallelism, but at the expense of a larger programming effort and a larger risk of deadlock. An alternative to mutual-exclusion-type locks for synchronization are nonblocking algorithms (lock-free, wait-free) [Gottlieb et al. 1983; Herlihy 1991]. These methods use atomic read-modify-write primitives, like CAS (compare and swap), that the hardware must provide. In contrast to locking, nonblocking programming allows threads to make progress even when other threads are delayed or blocked. However, the design of nonblocking algorithms is very hard and they have to be written using the available atomic primitives to achieve high performance. Transactional memory (TM) emerges as another alternative for nonblocking parallel programming [Herlihy and Moss 1993; Harris et al. 2010]. TM provides an abstraction, called transaction, that allows a thread to execute a sequence of accesses to shared memory in an atomic and isolated way.

TM offers simplicity in shared-memory multithreaded programming as it separates abstraction from implementation. Although TM was proposed almost two decades ago, its use as a parallel programming model in the context of high-performance computing is relatively new. Some works present a comprehensive performance evaluation of some TM implementations for synchronizing threads in parallel scientific applications, reporting little to modestly good performance benefits [Schindewolf et al. 2012; Bihari 2013; Yoo et al. 2013], and in sequential legacy codes [DeVuyst et al. 2011; Saad et al. 2012].

This article proposes TEPO (Transaction Execution under Precedence Order), a TMbased programming approach that offers the programmer both a parallel execution model defined to exploit maximum parallelism from transaction execution and a parallel programming model defined to hide from the programmer most of the complexities related to the analysis of data dependencies and explicit thread synchronization. The programmer focuses his or her attention on the partitioning of the program, or a section of the program (incremental parallelization), into a number of parallel chunks, and he or she may define a precedence execution order between them. This order is the one required to be fulfilled for the parallel program to be correct. Once the program has been partitioned, the parallel execution proceeds by running the chunks as transactions; that is, chunks execute optimistically in parallel as atomic blocks. However, in 
order to have a correct parallel execution, all transactions must update their chunk state according to the established precedence order.

The execution of the code chunks as transactions allows the underlying TM system to be in charge of tracking data dependencies and react when conflicts are detected that compromise the precedence execution order. We have designed a transactional manager based on decoupling transaction execution from transaction commit. Transaction execution is managed with the focus on exploiting parallelism, while transaction commits are managed to fulfill the defined precedence ordering.

Depending on how the programmer defines the precedence execution order, our model can be applied to parallelize sequential program sections or to improve the concurrency of existing multithreaded program sections. In addition, transactions make the TEPO parallel execution model independent of the specific implementation of the TM system.

The main contributions of this work are as follows:

-A transactional scheduler is developed that preserves a user-defined precedence execution order.

-A new transactional state (executed but not committed) is introduced to allow decoupling transaction execution from transaction commit.

- Based on the new transactional state, a relaxed commit condition is defined that allows, in some cases, transactions to commit out of the precedence order but fulfilling the sequential semantics of the original program.

- The TEPO model is applied to iterative code sections with total precedence execution order and linked with language-level directives.

\section{THE TEPO MODEL}

The goal of TEPO is to exploit parallelism from a sequential code section or to improve the concurrency of a multithreaded code section by resorting to a transactional memory engine to deal with data dependencies and chunk synchronization. TEPO comprises a transactional execution manager that tries to maximize parallelism exploitation while subjected to a transaction precedence order, and a parallel programming model defined to lessen programming effort.

The programmer should be concerned only about both the definition of the code chunks (program fragments that will be executed concurrently as transactions) and a precedence execution order relation between the chunks that sets the data dependencies between them. TEPO will be in charge of scheduling the execution of the code chunks as transactions keeping the precedence order with the aim of exploiting maximum concurrency. The precedence order is kept by TEPO by updating atomically the memory state of all executed chunks according to such order. TEPO is built on top of a transactional memory engine that takes care of detecting and solving memory conflicts between chunks.

\subsection{Code Chunks and Precedence Order}

Both concerns, code chunks and precedence order, have an important impact on the obtained parallel performance. If the chunks are not properly defined, they may exhibit many memory conflicts, resulting in an optimistic execution that may be drastically serialized. Or, the precedence order permits one to determine the type of data dependence that corresponds to a certain transactional conflict and decide which conflict resolution policy is best that hurts the performance the least.

There are different options to define a precedence execution order for transactions. Taking a sequential program section, we have (1) lexicographic order, which corresponds to the sequential order of instructions written by the programmer in the source code (concurrent transactions have to fulfill this order to ensure the sequential 
semantics of the original program); (2) dataflow order, a less restrictive order that corresponds to the order forced by true (flow) data dependencies between memory operations (the type of data dependence is derived from the lexicographic order); and (3) semantic order, an even less restrictive order that corresponds to the order forced by data dependencies existing in the problem (some flow data dependencies may be violated without generating an incorrect result).

As an example, if the programmer has defined single iterations of a DOALL loop as the code chunks, the lexicographic precedence order is given by the index variable of the loop, while, however, there is no dataflow precedence order (independent iterations). In the case of a scalar reduction loop, on the other hand, lexicographic and dataflow orders are the same, but there is no semantic precedence order (the final reduced result is independent of the execution order of the iterations due to the commutative and transitive properties of the reduction operator).

The programmer must decide which precedence execution order should be taken into account. If he or she wants to parallelize a sequential program section, the simplest option is the lexicographic order, as no data dependence analysis is required. Semantic information about the problem can also be used by the programmer to lessen the precedence restrictions. For instance, he or she can declare that the sequential code, for example, a loop, include reduction operations on some variables. On the other hand, there is a class of applications (some graph algorithms, image segmentation techniques, clustering algorithms, etc.) where the violation of some flow data dependencies does not result in an incorrect parallel version, but in a different but valid solution. For such applications, the programmer can choose different order possibilities.

The following definitions formalize the concepts described earlier. These definitions are intended to state the TEPO programming model.

Definition 2.1. A code chunk is a program execution unit into which a program code section is partitioned and can be executed within a transaction. The set of chunks for a code section $R$ is represented by $U=\left\{u_{0}, u_{1}, u_{2}, \ldots\right\}$.

The concept of chunk is commonly used in the TM context [Qian et al. 2012] as atomic blocks of instructions. These works assume that a core continuously executes chunks. However, we consider that chunk execution is defined only for one or several code regions of interest in the program.

Once the set $U$ is stated, the programmer can define a precedence order between chunks, as pointed out in the following two definitions.

Definition 2.2. The immediate precedence order relation $\left(\prec_{i}\right)$ is the order defined by the programmer according to some criterion, considering that a chunk never precedes itself.

Definition 2.3. The precedence order relation $(\prec)$ is the transitive closure of the immediate precedence:

$u, v \in U, u \prec v \Longleftrightarrow u \prec_{i} v$, or $\exists a_{0}, a_{1}, \ldots a_{n} \in U \mid u \prec_{i} a_{0}, a_{0} \prec_{i} a_{1}, \ldots, a_{n-1} \prec_{i} a_{n}, a_{n} \prec_{i} v$.

Note that a valid precedence relation $(\prec)$ must be, at least, a strict partial order relation; that is, it must be irreflexive, asymmetric, and transitive. This implies that $\prec_{i}$ must be free of cycles.

\subsection{Transactional Execution Model}

Our proposal is a chunk execution and scheduling model that extends a TM system to fulfill a precedence relation order defined by the programmer. Two major features of this model are as follows: (1) the execution of two chunks subjected to the precedence order is sequentially consistent; that is, the parallel execution is equivalent to a sequential 
execution of both chunks, one after the other (according to the precedence order); and (2) the chunks are executed atomically and in an isolated way by relying on the base TM conflict and versioning management.

The precedence relation is aimed at two objectives. First, it can help to parallelize a sequential code region. With no knowledge about the problem, the programmer can define a precedence order equivalent to the lexicographic order, ensuring the correctness of the parallel execution. However, concurrency can be improved if the programmer loosens precedence restrictions using problem dataflow and/or semantic information.

Second, it can be used to improve the exploited concurrency of a parallel code region. Our model utilizes the precedence order relation to make the base TM conflict resolution policy more efficient. This way, by means of the precedence order, the programmer can interact with the conflict resolution mechanism.

In order to proceed with the execution of a selected code section under the TEPO system, the following must be defined: (1) how the code section is partitioned into chunks, (2) the precedence order graph between chunks, (3) the pool of threads in charge of executing chunks, and (4) the function that maps chunks onto threads.

Definition 2.4. The mapping function, o, assigns chunks to threads, so $o: U \rightarrow T, T$ being the set of threads, $T=\{0,1 \ldots n$ Threads $\}$ (nThreads is the cardinality of the set of threads).

Hereafter, the notation $U_{t}$ will be used to describe those chunks in a set $U$ mapped to the thread $t$, that is, $U_{t}=\{u \in U \mid o(u)=t\}$.

Once all of this is stated, TEPO executes concurrently the chunks as ordinary transactions but with two main differences: (1) the conflict manager takes advantage of the precedence order to optimize the resolution of conflicts, and (2) the chunks are scheduled into threads according to the precedence order graph. Both issues will be discussed in detail in Sections 2.3 and 2.4.

Each thread starts executing the corresponding mapped chunks according to the precedence order. From its mapped set, the thread first selects one of the not-yetexecuted chunks with the lowest precedence order. Then, as the chunk executes transactionally, the base TM system records the memory addresses read and written (Read Set, $R S$, and Write Set, WS) required to ensure atomicity and isolation. Consequently, the TM system watches for data conflicts between concurrent chunks being executed by the pool of threads. If a conflict is eagerly detected, the conflict manager decides what to do to solve the situation, typically by aborting one of the transactions.

In addition to recording memory addresses, the TM system must also handle the updates to memory (version management). TEPO manages lazily the memory updates by buffering the transactional writes in special storage (Write Buffer) while keeping in memory the original data. However, in a TM system with strong atomicity, an eager version management is also possible.

When a thread finishes the execution of a chunk, it checks if the chunk can commit (see Section 2.3 for commit condition), as the precedence order has to be fulfilled. If the chunk is committed, the thread starts immediately a new chunk selected from the mapped set according to the precedence order. Otherwise, the chunk is kept in an executed but not committed state, a checkpoint of the chunk state (Write Buffer contents) is carried out, and the thread starts the execution of a new chunk (selected according to the precedence order). The Read Set and Write Set of the checkpointed chunk remain active because its data updates are still noncommitted, so data conflicts with other running chunks (from the same or different threads) are possible.

Under the TEPO execution model, the chunks are executed as transactions and can be in one of the states described in Figure 1. In contrast to conventional TM systems, 


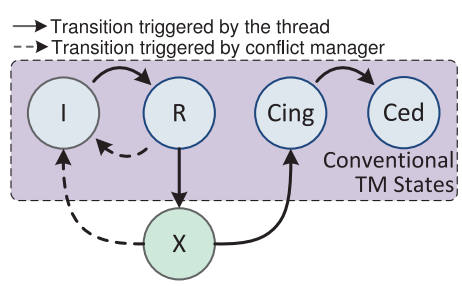

\begin{tabular}{|l|l|}
\hline State & Description \\
\hline \hline I (Idle) & The chunk is waiting to be executed \\
\hline R (Running) & The chunk is running \\
\hline X (eXecuted) & The chunk is executed and ready to be committed \\
\hline Cing (Committing) & The chunk is being committed \\
\hline Ced (Committed) & The chunk is committed \\
\hline
\end{tabular}

Fig. 1. Chunk state diagram.

in TEPO the end of the execution of a chunk and its commit are uncoupled states. An executed chunk can commit immediately or be put aside (checkpointed) so the thread is free to start a new chunk. The thread is in charge of determining periodically if pending chunks can commit or not at some later point.

Figure 1 shows the chunk state diagram that describes the behavior of TEPO. All chunks mapped to a thread are initially in the $I$ (idle) state. At a given time, the thread is executing one of its chunks, which is in the $R$ (running) state. When that chunk finishes its execution, it transits to the $X$ state and the thread checks the commit condition (see next section). If it is fulfilled, the thread starts committing the chunk (Cing state) that eventually becomes committed (Ced state). If the commit condition fails, the chunk remains in the $X$ (executed but not committed) state and the thread starts a new chunk. Every time the execution of a chunk is finished, the thread checks again the commit condition for all $X$-stated chunks (in the precedence order), committing those that fulfill the condition. Note that the thread is in charge of upgrading the state of the chunk (from idle to committed, state by state), while the conflict manager can downgrade the state if a data conflict is detected. The state diagram assumes that a conflict is solved by aborting the chunk (going to the idle state).

\subsection{Conflict Management}

Conflict management is composed of two operations: conflict detection and conflict resolution. Conflict detection refers to the detection of data conflicts between running ( $R$ state) and/or executed ( $X$ state) chunks, mapped to the same or different threads. Conflict resolution refers to the action taken to solve the conflict (ensure chunk atomicity). In TEPO, the base TM system performs both operations, detection and resolution, eagerly, that is, as soon as a chunk tries to access a conflicting memory location.

The common action to solve a data conflict consists of aborting one of the offending chunks (going to $I$ state) and restarting it. Usually, the abort action has an important impact on performance as all work done by the aborted chunk is lost and re-executed again. To reduce this negative impact and improve chunk concurrency, TEPO takes advantage of the precedence order relation defined in the set of chunks to introduce new actions: stalling and data forwarding.

In order to describe the TEPO conflict resolution, the following sets are defined.

Definition 2.5. Denoting by $U_{S}$ the set of all chunks in state $S$ (being $S$ one of $I, R$, $X$, Cing, or Ced) across all threads, we define the sets of precedent, subsequent, and nonrelated chunks of a given chunk as

$$
\begin{aligned}
& \operatorname{prev}_{S}(u)=\left\{v \in U_{S} \mid v \neq u, v \prec u\right\} \\
& \operatorname{next}_{S}(u)=\left\{v \in U_{S} \mid v \neq u, u \prec v\right\} \\
& \operatorname{norel}_{S}(u)=\left\{v \in U_{S} \mid v \neq u, u \nprec v, v \nprec u\right\} \\
& \operatorname{prev}(u)=\bigcup_{U_{S}} \operatorname{prev}_{S}(u) ; \operatorname{next}(u)=\bigcup_{U_{S}} \operatorname{next}_{S}(u) ; \operatorname{norel}(u)=\bigcup_{U_{S}} \operatorname{norel}_{S}(u) .
\end{aligned}
$$


Table I. Conflict Resolution in TEPO: Thread $t$ Is Running the Chunk $u$ That Accesses the Memory Location $m$ in a Given Time; $v$ ls Other Chunk in $U, v \neq u$, That Accessed $m$ Previously $(R S(v)$ : Read Set, WS(v): Write Set)

\begin{tabular}{|c|c|c|c|c|}
\hline & \multicolumn{2}{|r|}{$u$ reads $m$} & \multicolumn{2}{|c|}{$u$ writes $m$} \\
\hline & $m \in R S(v)$ & $m \in W S(v)$ & $m \in R S(v)$ & $m \in W S(v)$ \\
\hline$\forall v \in \operatorname{norel}_{R}(u)$ & No action & (1) Abort $u$ & (1) Abort $u$ & (1) Abort $u$ \\
\hline$\forall v \in \operatorname{prev}_{R}(u)$ & No action & $\begin{array}{c}\text { (2) Abort } u, \\
\text { Stall } u, \text { Forward } v \rightarrow u\end{array}$ & (3) No action & (4) Commit $v$ before $u$ \\
\hline$\forall v \in \operatorname{next}_{R}(u)$ & No action & (5) No action & (6) Abort $v$ & (4) Commit $u$ before $v$ \\
\hline$\forall v \in \operatorname{norel}_{X}(u)(o(v) \neq t)$ & No action & (1) Abort $u$ & (1) Abort $u$ & (1) Abort $u$ \\
\hline$\forall v \in \operatorname{norel}_{X}(u)(o(v)=t)$ & No action & (7) Forward $v \rightarrow u$ & (7) No action & (7) Commit $v$ before $u$ \\
\hline$\forall v \in \operatorname{prev}_{X}(u)(o(v) \neq t)$ & No action & $\begin{array}{c}\text { (2) Abort } u, \\
\text { Stall } u, \text { Forward } v \rightarrow u\end{array}$ & (3) No action & (4) Commit $v$ before $u$ \\
\hline$\forall v \in \operatorname{prev}_{X}(u)(o(v)=t)$ & No action & (2) Forward $v \rightarrow u$ & (3) No action & (4) Commit $v$ before $u$ \\
\hline$\forall v \in \operatorname{next}_{X}(u)(o(v) \neq t)$ & No action & (5) No action & (6) Abort $v$ & (4) Commit $u$ before $v$ \\
\hline$\forall v \in \operatorname{next}_{X}(u)(o(v)=t)$ & Not possible & Not possible & Not possible & Not possible \\
\hline$\forall v \in \operatorname{norel}_{\text {Cing }}(u)(o(v) \neq t)$ & No action & $\begin{array}{c}\text { Abort } u, \text { Stall } u \\
\text { Forward } v \rightarrow u, \text { Read }^{*} m\end{array}$ & Abort $u$, Stall $u$ & Abort $u$, Stall $u$ \\
\hline$\forall v \in \operatorname{norel}_{\text {Cing }}(u)(o(v)=t)$ & Not possible & Not possible & Not possible & Not possible \\
\hline$\forall v \in \operatorname{prev}_{\text {Cing }}(u)(o(v) \neq t)$ & No action & $\begin{array}{c}\text { (2) Abort } u \text {, Stall } u \\
\text { Forward } v \rightarrow u \text {, Read* } m\end{array}$ & (3) No action & $\begin{array}{c}\text { (4) Stall } u \text {, } \\
\text { Commit } v \text { before } u\end{array}$ \\
\hline$\forall v \in \operatorname{prev}_{\text {Cing }}(u)(o(v)=t)$ & Not possible & Not possible & Not possible & Not possible \\
\hline$\forall v \in \operatorname{next}_{\text {Cing }}(u)$ & Not possible & Not possible & Not possible & Not possible \\
\hline
\end{tabular}

${ }^{*}$ Only if $m$ already updated in memory (Cing atomic address by address).

Being consistent with Definition 2.4, the notation $\operatorname{norel}_{S}(u)_{t}=\operatorname{norel}_{S_{t}}(u)$ restricts the set for those chunks owned by thread $t$. The same is applicable to $\operatorname{prev}()$ and next() functions.

Table I shows the actions that the TEPO conflict resolution policy performs when a data conflict is detected eagerly. In some cases, the policy can choose one in several possible actions. The actions are defined with the aim of maximizing concurrency in the execution of chunks in different threads.

The conflict resolution policy depends on the commit condition for chunks subjected to a precedence order relation. We define a strict commit condition as follows.

Definition 2.6. Concurrent chunks subjected to a precedence order $(\prec)$ fulfill the strict commit condition if: Given $u \in U, u$ in state $X, u$ transits to state $\operatorname{Cing}$ if $\forall v \in U$, $v \prec u, v$ is in state $C e d$.

All actions with label (1) in Table I correspond to the default behavior of the base TM system (that is, nonordered chunks being executed in different threads). For simplicity, we assumed the default abort action. Actions with label (2) correspond to data conflicts when chunk $u$ reads $m$, which was previously written by chunk $v$ and $v \prec u$ (RAW dependence). In this case, the commit condition ensures that $u$ will commit later than $v$. So, this conflict can be solved by aborting/stalling $u$ or forwarding the updated data from $v$ to $u$. However, if both chunks are mapped to the same thread, the only possible action is data forwarding because, otherwise, the thread will be deadlocked. On the other hand, if $v$ is committing, it is possible for $u$ to read data in memory if the update is already there (considering a nonatomic Cing state).

Actions with label (3) correspond to a WAR dependence ( $u$ writes and $v$ reads, and $v$ must commit before $u$ ). The commit condition permits filtering out these conflicts (no action), as concurrent execution is always correct. Actions with label (4) correspond to a WAW dependence ( $u$ and $v$ write in $m$ and one chunk precedes the other chunk). 


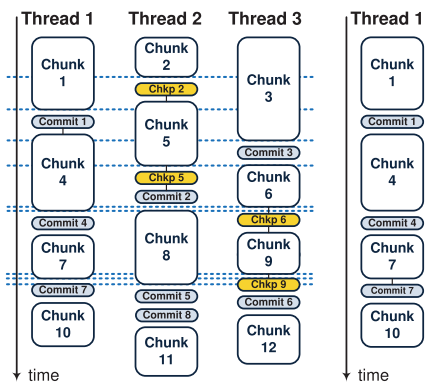

(a)

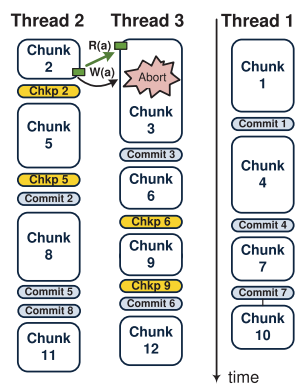

(b)

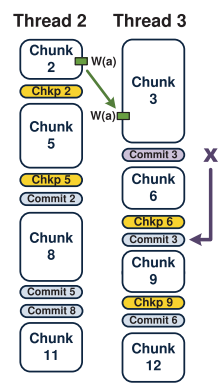

(c)

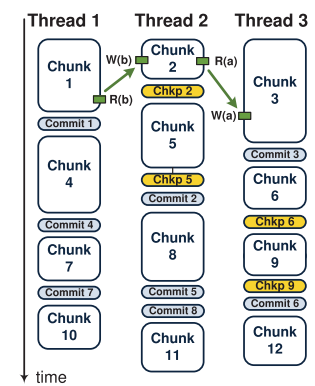

(d)

Fig. 2. Some TEPO execution scenarios (total order relation): (a) out-of-order commits (relaxed commit condition, (b) RAW dependence (case (6) in Table I), (c) WAW dependence (case (4) in Table I), and (d) WAR dependencies (cases (5) and (3) in Table I).

The commit condition ensures that the correct written data is the last that updates memory.

Actions with label (5) correspond also to a WAR dependence because the reading chunk $(u)$ must commit first. So, these conflicts are solved similarly to the case (3). Actions with label (6) correspond to a RAW dependence, as the writing chunk $(u)$ must commit first. In these cases, as the read in $v$ was performed earlier in time than the write in $u$, the only possible action is aborting $v$. Finally, actions with label (7) correspond to nonrelated chunks mapped to the same thread. By default, they commit in the same order as they are executed.

From Table I it can be seen that an executed chunk $v$ ( $v$ in state $X$ ) can be aborted only by a preceding and conflicting running chunk $u$ ( $u$ in state $R, u \prec v$ ). This result allows one to relax the commit condition.

Definition 2.7. Concurrent chunks subjected to a precedence order $(\prec)$ fulfill the relaxed commit condition if: Given $u \in U, u$ in state $X, u$ transits to state Cing if $\forall v \in U, v \prec u$ and $W S(u) \cap W S(v)=\emptyset, v$ is in state either $X$, Cing, or Ced.

This relaxed condition permits one to perform out-of-order commits (regarding precedence order) and, consequently, improve concurrency between chunks in some situations. Figure 2 shows an example scenario of three threads executing 12 chunks subjected to a total precedence order relation. ${ }^{1}$ Figure 2(a) illustrates out-of-order commits thanks to the application of the relaxed commit condition. Chunk 3 commits before chunk 2 because chunk 1 is already committed and chunk 2 is already executed (state $X)$. Note that the relaxed commit condition cannot be applied, however, to transactions subjected to a WAW dependence, as shown in the last column of Table I. Figure 2 also shows an example of a (b) RAW conflict corresponding to the case (6) in the third row of Table I, a (c) WAW conflict that corresponds to the case (4) in the same table (sixth row), and (d) WAR conflicts corresponding to cases (5) and (3) in Table I (eighth and sixth rows, respectively).

\subsection{Transaction Scheduling}

The TEPO scheduler is in charge of scheduling and executing, as transactions, the chunks mapped to threads, as well as committing those threads that fulfill the commit condition (strict or relaxed). The commit condition is defined so the precedence order relation over chunks is preserved, as stated in the following definition.

\footnotetext{
${ }^{1}$ That is, an order relation where totality is satisfied: $\forall a \neq b$, either $a \prec b$, or $b \prec a$.
} 
Table II. TEPO Scheduling: Transactional Primitives

\begin{tabular}{|l|c|l|}
\hline Primitive & State Transition & \multicolumn{1}{c|}{ Action } \\
\hline \hline TX_begin() & $I \rightarrow R$ & Allocate new RS, WS, WB \\
\hline TX_checkpoint() & $R \rightarrow X$ & Save current RS, WS, WB \\
\hline TX_commit_check() & - & Check commit condition \\
\hline TX_commit() & $X \rightarrow$ Cing $\rightarrow$ Ced & $\begin{array}{l}\text { Commit chunk, deallocate } \\
\text { RS, WS, WB }\end{array}$ \\
\hline
\end{tabular}

RS: Read Set; WS: Write Set; WB: Write Buffer.

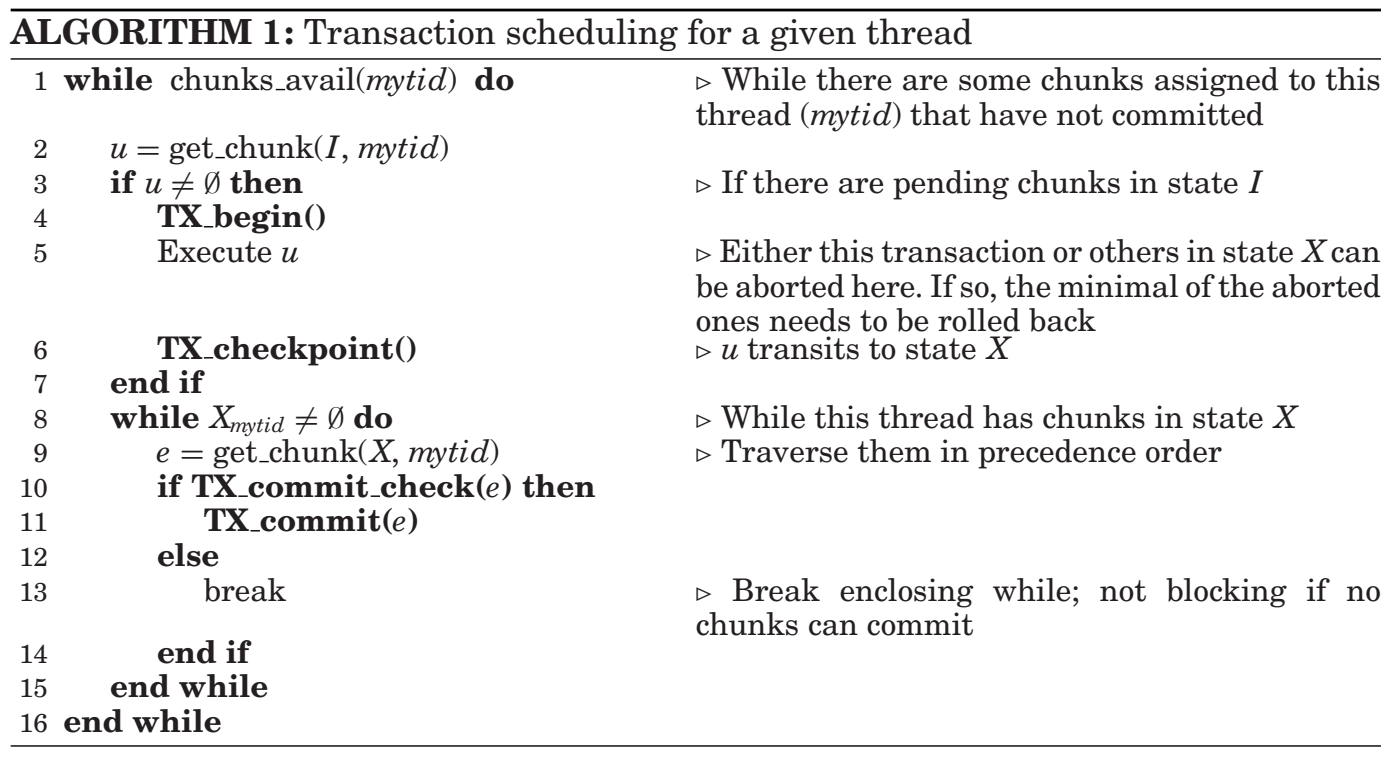

Definition 2.8. The chunk scheduling bounded by a precedence order is defined as:

(1) Intrathread scheduling: Those chunks mapped to the same thread start their execution according to the precedence order; that is, if $u, v \in U_{t}$ and $u \prec v$, then the thread $t$ starts the execution of $u$ earlier than $v$.

(2) Interthread scheduling: Those chunks mapped to different threads commit their execution as soon as possible according to the commit condition (strict or relaxed).

Our scheduling model aims at improving the exploited concurrency in two ways: intrathread and interthread. The first one is improved by allowing a thread to have several pending executed chunks waiting for commit while executing another chunk, avoiding the stalling of the thread this way. This is the main benefit of intrathread concurrency. On the other hand, thanks to the relaxed commit condition, nonconflicting chunks executed by different threads may commit out of order. This is the main benefit of interthread concurrency.

TEPO transaction scheduling is a fully distributed operation (see implementation in Section 4) designed to minimize scheduling overhead and synchronization and maximize chunk execution concurrency. This operation was outlined at the end of Section 2.2, and it is described in detail in Algorithm 1 together with Tables II and III.

\section{APPLICATION TO ITERATIVE CODES}

This section analyzes how iterative codes (i.e., loops) can benefit from the TEPO system and the key aspects of its implementation. In iterative codes, the precedence order is a total order relation. The aim is maximizing concurrency when implementing 
Table III. TEPO Scheduling: Helper Functions

\begin{tabular}{|l|l|}
\hline Function & \multicolumn{1}{c|}{ Meaning } \\
\hline chunks_avail $(t)$ & $\begin{array}{l}\text { Return false if all mapped } \\
\text { chunks to } t \text { are committed; } \\
\text { otherwise, return true }\end{array}$ \\
\hline get_chunk $(A, t)$ & $\begin{array}{l}\text { Return a minimal* chunk in } \\
\text { state } A \text { for thread } t \text {; if several } \\
\text { minimals are available, } \\
\text { select one arbitrarily; } \\
\text { otherwise, return } \varnothing\end{array}$ \\
\hline
\end{tabular}

${ }^{*}$ Chunk $a \in Y$ is minimal if $\nexists b \in Y / b \prec a$.

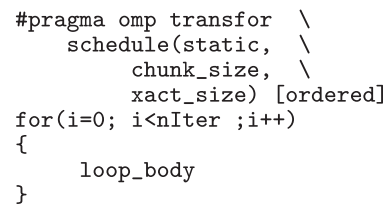

Precedence order: $u_{k} \prec_{i} u_{k+1}$

Mapping: $o\left(u_{k}\right)=k \bmod n$ Threads

Fig. 3. The loop is partitioned into chunks, with a total (sequential) precedence order.

transactional iterative constructs, such as the transfor block proposed by the OpenTM API [Baek et al. 2007], shown in Figure 3. According to its definition, the transf or construct can be tuned by means of three parameters: the loop scheduling, ${ }^{2}$ the chunk size (iterations assigned to each thread on every scheduling decision), and the transaction size (number of loop iterations executed per transaction). For the sake of simplicity, in what follows, the chunk size will be considered equal to the transaction size for the static scheduling (chunkSz).

In order to express a transf or block in terms of the framework presented in Section 2 , it is necessary to define three parameters: how to partition the code into chunks, the precedence relation between such chunks, and the function that maps chunks onto threads. A simple partition is to divide the iteration space into chunks of chunkSz contiguous iterations: $L=\left\{u_{0}, u_{1}, \ldots\right\}$. On being considered as a sequence, chunks are subjected to the following immediate precedence relation: $u_{0} \prec_{i} u_{1} \prec_{i} u_{2} \ldots$. The static loop scheduling corresponds to map chunks to threads through a block-cyclic mapping function: $o\left(u_{i}\right)=i \bmod n T h r e a d s$.

A key aspect of TEPO is that the precedence order defined by the programmer is preserved through commits for these iterations with dependencies. The ordered clause denotes this feature. Note that even if the loop were fully parallel (DOALL loop), commits would take place chunk by chunk in the precedence sequential order. However, when precedence order between iterations is not a concern (e.g., when iterations can be arbitrarily reordered), our model allows chunks of iterations to commit as they are successfully executed without waiting for others. Simply, programmers have to omit the clause marking the execution in order. Nonetheless, in these cases, an artificial order has been introduced with the aim of simplifying the implementation, which can be the creation timestamp of transactions.

\section{IMPLEMENTATION}

In this section, details about the implementation of the TEPO model on a lightweight STM system is discussed. In particular, TinySTM [Felber et al. 2008] has been used as the baseline system that has been properly modified in order to introduce TEPO.

\footnotetext{
${ }^{2}$ OpenTM reuses the scheduling options defined by OpenMP. This work considers only the static scheduling, which is interpreted as a block-cyclic distribution of equal-sized chunks among threads.
} 
Table IV. Generic Notation

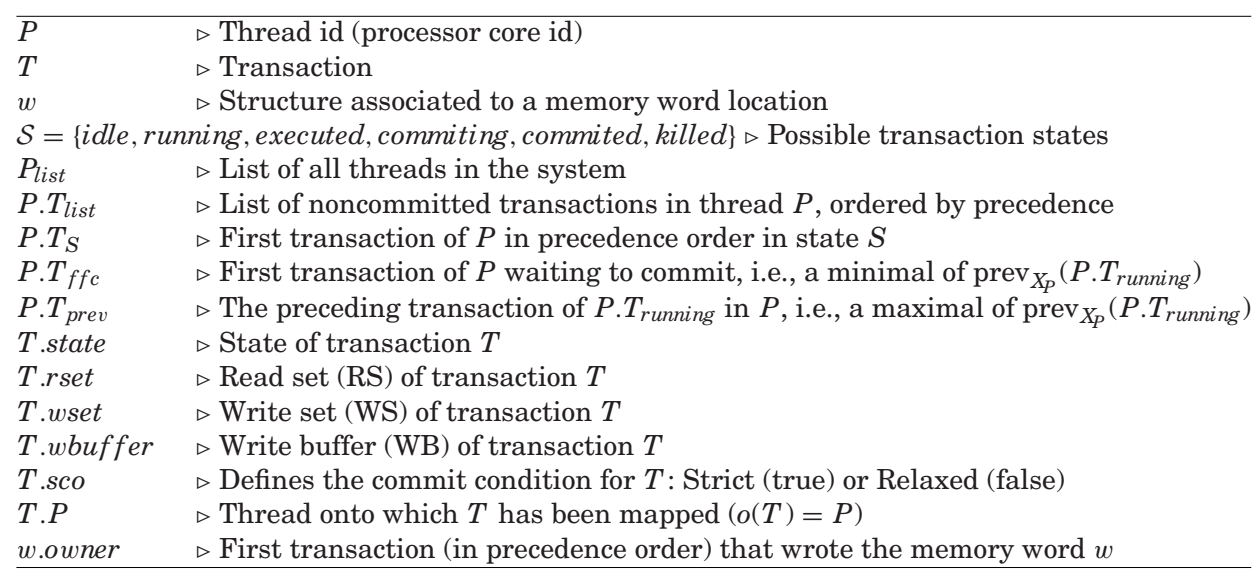

Table V. Generic Utility Functions

\begin{tabular}{|c|c|}
\hline$\overline{\operatorname{GETWORD}(m)}$ & $\triangleright$ Return a word structure for the memory address $m$ \\
\hline $\operatorname{GetFromWbuFF}(P, w)$ & $\begin{array}{l}\triangleright \text { Return the value of word } w \text { from the Write Buffer of the last } \\
\text { transaction writing } w \text { in } P\end{array}$ \\
\hline $\operatorname{ADDToWBufF}(P, w, v)$ & $\begin{array}{l}\triangleright \text { Insert/Update a value } v \text { into the } w \text { entry of the Write Buffer of the } \\
\text { running transaction }\end{array}$ \\
\hline $\operatorname{GETFROMMEMORY}(w)$ & $\triangleright$ Return value of word $w$ from Memory \\
\hline $\operatorname{COPYTOMEMORY}(T)$ & $\triangleright$ Dump the Write Buffer of a transaction to Memory \\
\hline $\operatorname{ADDToWsET}(P, w)$ & $\triangleright$ Insert/Update an entry into the Write Set of the running transaction \\
\hline $\operatorname{ADDToRseT}(P, w)$ & $\triangleright$ Insert/Update an entry into the Read Set of the running transaction \\
\hline RESTOREPC $(T)$ & $\triangleright$ Restore the Program Context at the beginning of $T$ \\
\hline CHECKCOMmitCondition $(T)$ & $\triangleright$ Check if the transaction $T$ can be committed safely \\
\hline $\operatorname{EXISTFREESLOT~}(P)$ & $\triangleright$ True if a free Slot is available to start a new transaction \\
\hline $\operatorname{NEW}(T)$ & $\begin{array}{l}\triangleright \text { Get resources for a new transaction } T \text {, allocating a new Slot and its } \\
\text { associated Data Sets and Write Buffer; program context of the } \\
\text { transaction entry point needs to be saved }\end{array}$ \\
\hline $\operatorname{RELEASE}(T)$ & $\triangleright$ Release all resources associated to $T$ (Data Sets, Write Buffer, Slot) \\
\hline
\end{tabular}

The baseline configuration considers an encounter-time locking (ETL), for which the conflicts of the running transactions are detected early with a write-back policy; that is, a Write Buffer (redo-log) is defined to buffer the write operations and copy new values in memory lazily upon commit. Furthermore, although approaches such as stalling and data forwarding have been proposed as two feasible TEPO conflict resolution policies, in order to simplify the implementation, the conflict resolution only considers the abort operation. Most of the base transactional primitives, such as read, write, rollback, and commit, need to be adapted as described next.

Following a similar notation to that found in Riegel et al. [2007], Algorithms 4 through 7 describe the adapted core transactional primitives. Some general notation and several utility functions are defined in Tables IV and V. In this description, the term transaction is adopted as being equivalent to chunk, which has been used in previous sections, insomuch that a chunk represents a portion of the program mapped as an executable unit into a transaction.

It will be assumed that only one single thread is attached to a processor core, so that the variable $P$ refers to the thread identifier. A data structure per thread, the pool of Slots, is defined to allow multiple active transactions. Each Slot references the Read Set, the Write Set, and the Write Buffer for each uncommitted transaction. At least one of these Slots is active in a given moment (associated to the running transaction). 


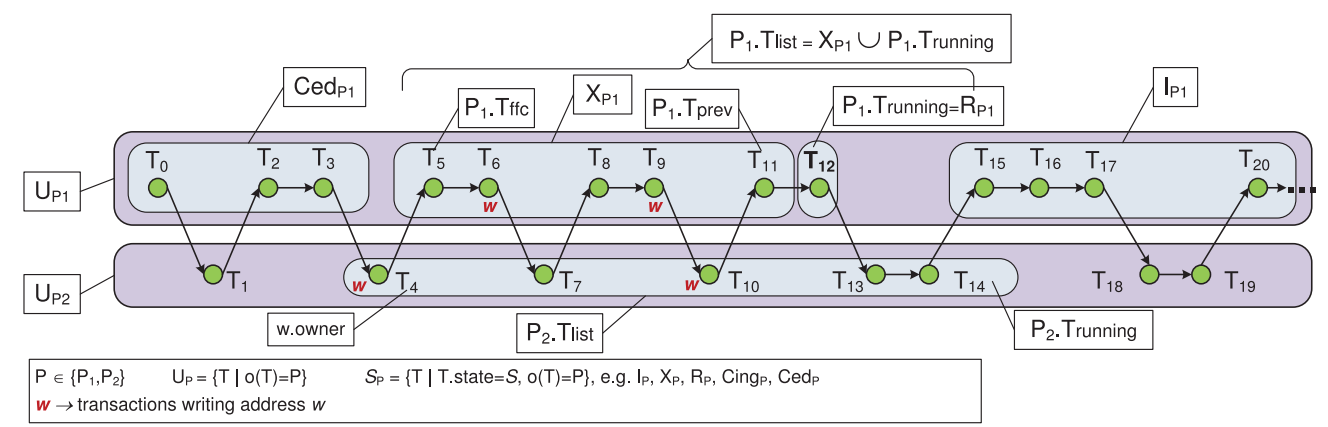

Fig. 4. TEPO implementation: a set of transactions is mapped onto two threads. Arrows represent immediate precedence order in a Hasse-like style. While no data conflict exists, transactions can progress forward. Running transactions can conflict with either running or executed-but-not-committed transactions of other threads. For each thread, two remarkable transactions are the running one and the first waiting for the committing one (minimal of those in state $X$ ).

The pool of Slots is described in Section 4.4. The Write Buffer could play the role of the Write Set, but in order to be more general, both of them are kept separate, as other configurations different from ETL could not use a Write Buffer. Together with the transaction states declared in Section 2.2, an auxiliary transitional state killed is introduced, indicating that a given transaction is marked as aborted and consequently needs to be restarted suitably when checked later. For the sake of clearness, a total precedence order (pure iterative) is assumed in what follows. In Figure 4, this kind of scenario is depicted for two threads and a set of transactions with a total (iterative) precedence order. Transactions of interest have been marked in the figure according to the notation introduced here and in Section 2.

\subsection{Read}

Algorithm 4 corresponds to the transactional Read primitive. In contrast to the baseline implementation, Read starts checking if there is any precedent (according to $\prec$ ) executed but not committed or running transaction that has been killed (aborted) by another precedent one. These aborted (killed) transactions must be restarted. Note that this step is not needed in the baseline implementation because only one transaction exists per thread. The next step is checking if the memory address has been written and where. The baseline implementation looks up the address only in its own Write Set. However, TEPO needs to look it up in all precedent transactions (running or executed but not committed). If none of the precedent transactions has written the requested address, the value can be loaded directly from memory. If it has been written by the current transaction, the value can be loaded from the Write Buffer. Otherwise, a precedent transaction wrote the address and consequently a data conflict is detected, aborting the current transaction. ${ }^{3}$ If read is successful, the memory address is finally included in the Read Set.

\subsection{Write}

Algorithm 5 shows the Write primitive pseudocode. Similar to the Read operation, Write starts checking the state of any executed but not committed or running transaction. If the Write Set of the current running transaction already contains the requested address, then this thread was the last one that wrote this location and the

\footnotetext{
${ }^{3}$ This conflict could be solved also by stalling the current transaction or by forwarding speculatively the requested data from the precedent owner transaction.
} 

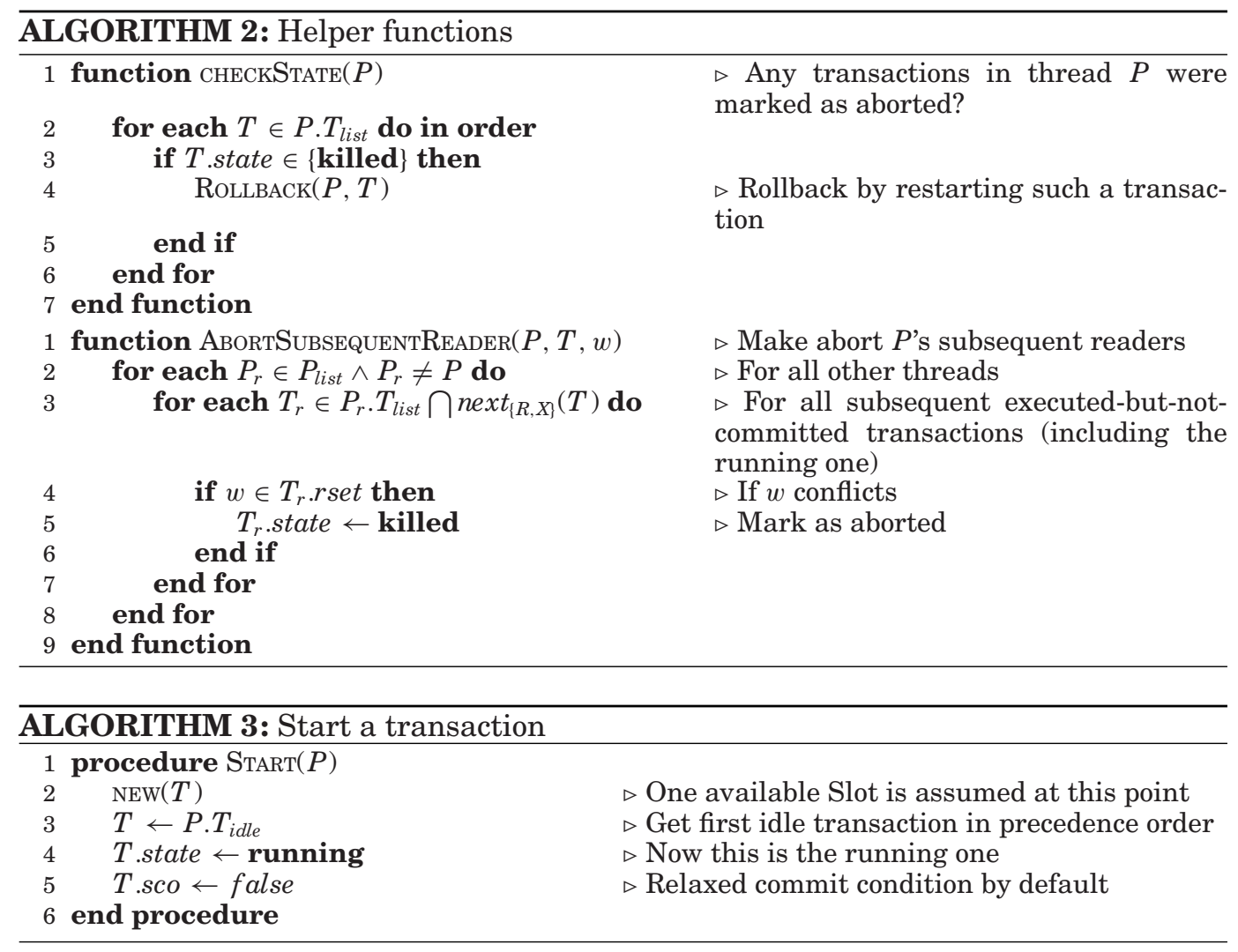

corresponding entry in the Write Buffer must be overwritten with the new value. If, instead, another thread has written this address (not in the local Write Set), a WAW conflict needs to be handled. As previously mentioned, those conflicts are solved by considering the precedence order criterion and forcing the commit of the conflicting transactions in strict sequential order. In this case, the SCO bit (Strict Commit Order bit) is asserted for the writer with the last place in precedence order. Transactions having the SCO bit active cannot commit until all previous (in precedence order) transactions have committed. In any case, either if the address was not written or if it was written by another transaction, a new entry needs to be allocated in the local Write Buffer with the new value, and similarly the address is inserted in the transaction's Write Set. Finally, the transactional Write operation needs to force an abort of all transactions that follow in precedence order and have read the same memory address. This last step is required because values having been read by subsequent transactions (that follow in precedence order) may have become inconsistent.

\subsection{Rollback}

The Rollback primitive, shown in Algorithm 6, allows the system to restore itself from an inconsistent state by aborting a conflicting transaction and restoring the previous safe program context. The rollback operation is faster in lazy version management systems (write-back) because data are not required to be restored into the memory. The process consists simply of flushing the Write Buffer and resetting both the Write Set and the Read Set. Then, the transaction can go back to the initial point and retry its execution. The TEPO rollback operation is very similar to that of the baseline 


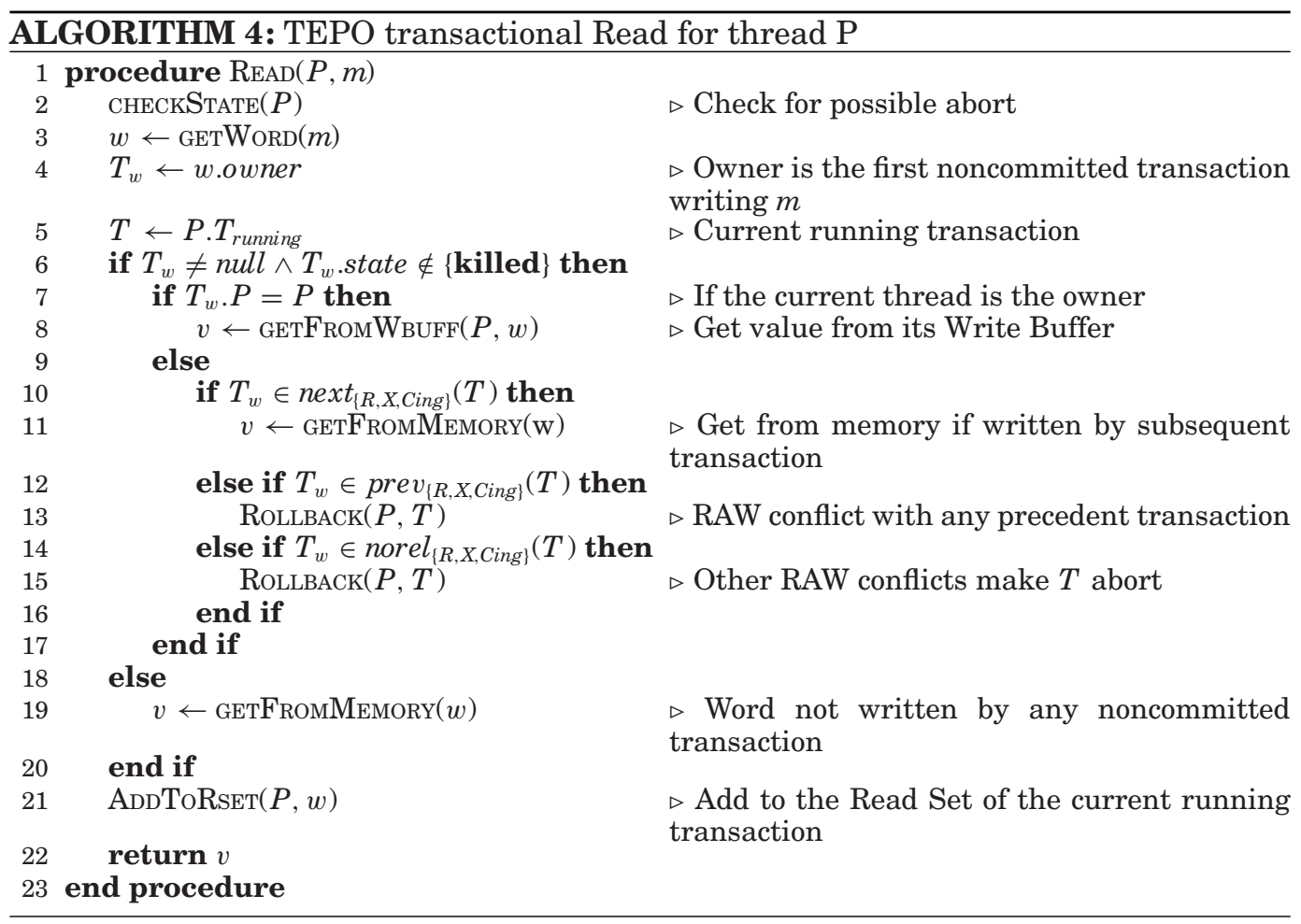

implementation. However, it is necessary to support multiple transactions per thread, apart from the current one. In this way, when a transaction aborts, all subsequent transactions belonging to this thread also need to abort. Therefore, starting from the current running transaction, the rollback process is repeated until the conflicting transaction is reached and the program context is restored to the beginning of this one.

\subsection{Commit}

Algorithm 7 shows a pseudocode for the commit phase. As it occurs in the Write and Read primitives, the Commit starts with a check for possible aborted transactions in the current thread. If none of them has aborted, the commit phase proceeds, starting with the first pending transaction in precedence order inside the committing thread. In order to verify the commit condition (Section 2.3) and fulfill the transaction precedence restrictions, a shared data structure is necessary to keep track of already committed transactions. For instance, this can be a bit vector. This shared data structure needs to be accessible by all threads and it needs to be acquired and written atomically by the committing thread. In the case of total order (pure iterative), a shared counter can be used, which will point to the last committed transaction [Gonzalez-Mesa et al. 2012]. Following the transaction precedence order, for each transaction to be committed, the Write Buffer contents need to be dumped to memory, and the Write Set and Read Set are reset. When a transaction does not fulfill the commit condition, no more transactions of this thread are committed.

Because there could be an unlimited number of transactions per thread being executed but not committed that have been checkpointed, the resources required for these checkpoints might grow up without restriction. In order to bound such an amount of resources (Write Buffer, datasets), a limited pool of Slots has been defined per thread 

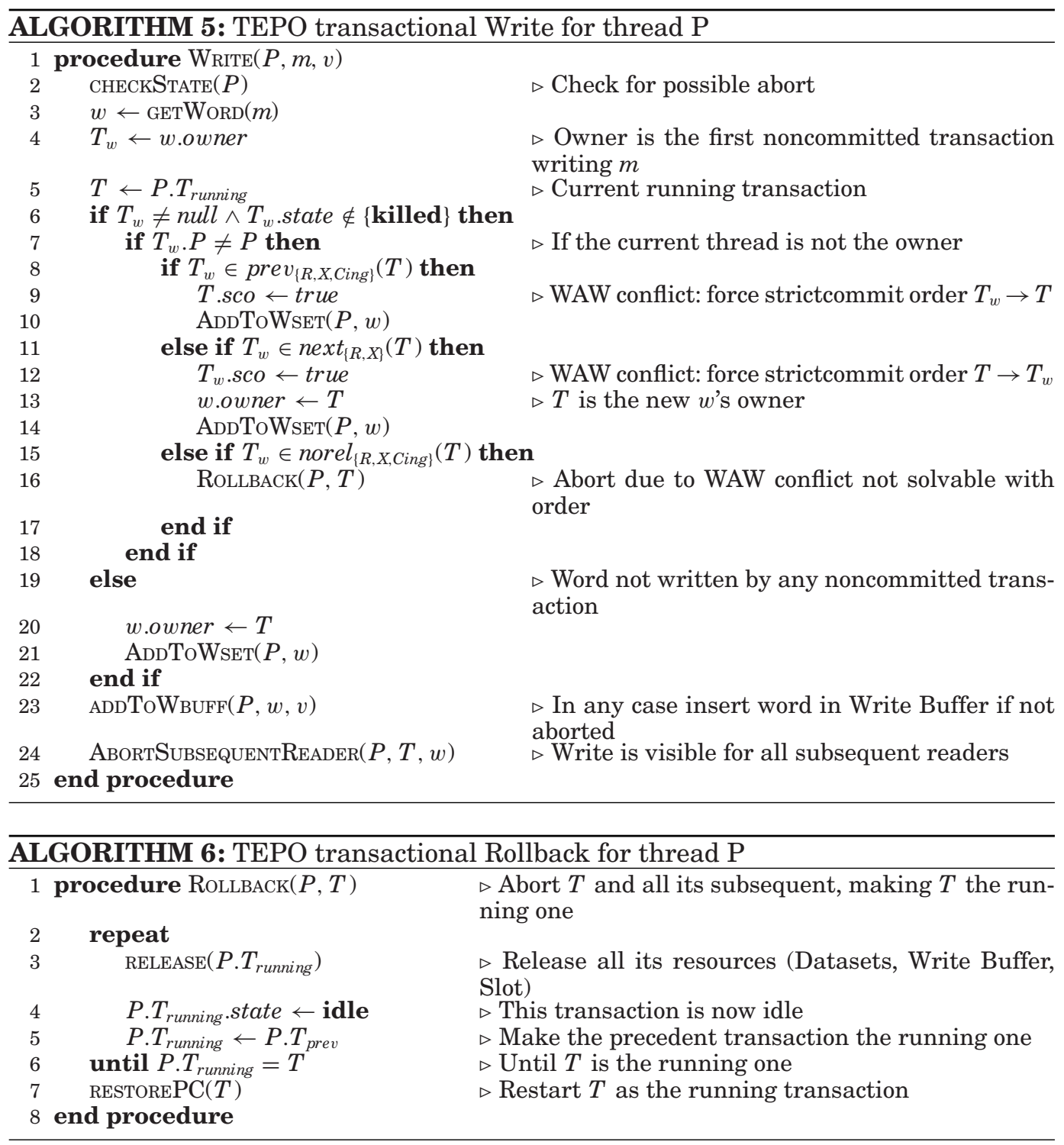

[Gonzalez-Mesa et al. 2012]. For a total precedence order, this pool has been implemented as a circular buffer (Figure 5) with a limited number of reusable Slots, large enough to allow several uncommitted transactions without increasing significantly the risk of overflow. A similar solution can be found in Ferri et al. [2011]. By means of two pointers, the Slot of the just committed transaction can be freed (next to commit pointer in Figure 5), or a new Slot can be elicited (next available pointer). Each Slot is associated to the Read Set, the Write Set, and the Write Buffer of their corresponding transaction, along with the program context of their transaction entry point. Implemented in this manner, an explicit checkpoint is not necessary, as indicated in Algorithm 1, because a new Slot is allocated when a new transaction starts. If the available Slots are exhausted, no new transaction can be launched by the thread. So, the thread stalls until one of the pending executed transactions is committed successfully. 

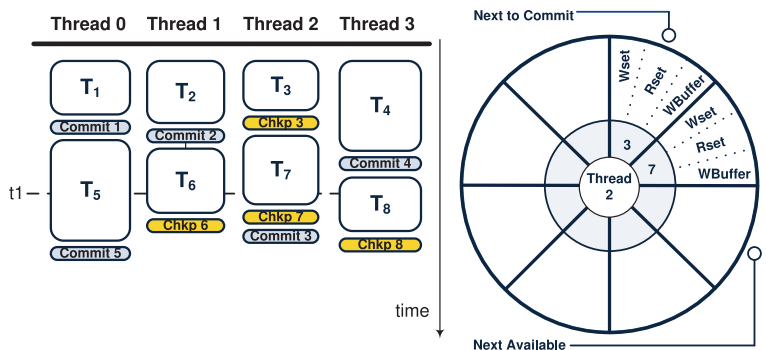

Fig. 5. A pool of Slots, implemented as a circular buffer, allow keeping multiple transactions per thread. Checkpointing is implicit by getting a new Slot.

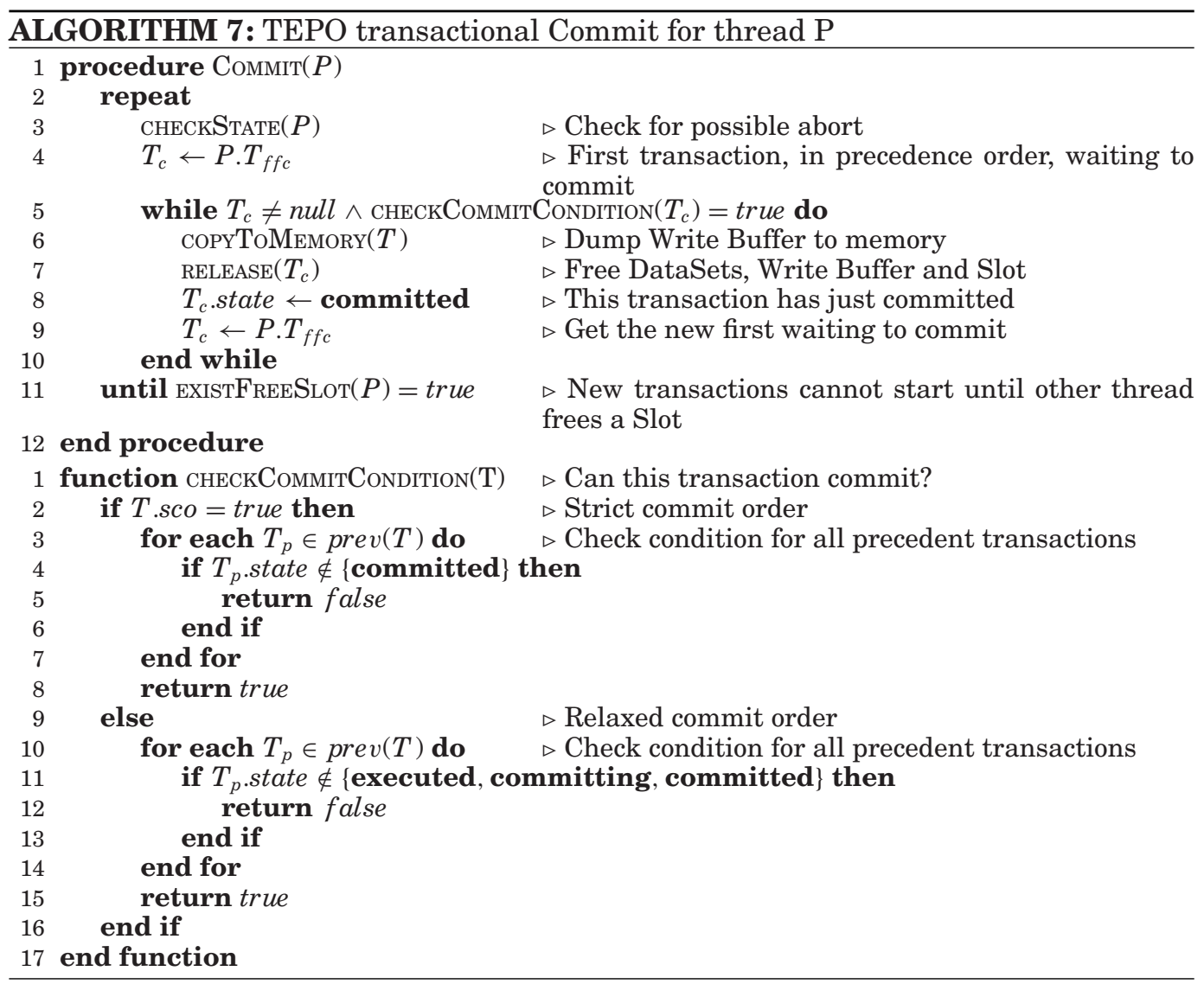

Note that the circular buffer is a particular implementation for iterative codes, where a total precedence order is preserved. In general, for a partial order, a more sophisticated free slot manager would be required.

In our experimental evaluation, the number of transactions per thread was well balanced, so the average number of uncommitted transactions per thread resulted around three. The size of the circular buffer in our experiments was set to eight entries. Observe that both datasets and the write buffer are dynamic structures whose size changes as the transaction runs. 


\section{EXPERIMENTAL EVALUATION}

The TEPO approach for loops implemented on top of TinySTM has been evaluated on a selection of benchmark codes. This section discusses the results of this evaluation. Experiments were conducted on a quad-socket eight-core Intel Xeon X7550 multiprocessor (32 cores) at $2 \mathrm{GHz}$, running Linux kernel 2.6.32 (64-bit). Codes were compiled using gcc version 4.3.4, with optimization option - $\mathrm{O} 2$ for the transactional library (as recommended by TinySTM) and optimization -O3 for the benchmark codes.

Three performance tests have been carried out. First, the transactional benchmark suite STAMP [Cao Minh et al. 2008] has been used as a reference, in order to compare TEPO against the baseline TinySTM. Second, the ability for exploiting concurrency of TEPO has been evaluated on two irregular iterative codes: Sparse Matrix Transposition [Pissanetzky 1984] and Fluidanimate [Bienia et al. 2008], a code including histogram reduction loops. Finally, with the aim of measuring overheads introduced by TEPO with respect to the baseline STM software, both implementations have been evaluated using two versions of an iterative 2D stencil code, Jacobi and Seidel from Polybench [Pouchet 2011]. In all of these benchmarks (except STAMP), transactions have been manually created by strip-mining the original loop. The new inner loop, which runs over a block of iterations, is encapsulated in a chunk and executed as a transaction. In Jacobi, Seidel, and Sparse Matrix Transposition, the loop that iterates over rows is the one partitioned into chunks. In Fluidanimate, the partitioned loop is the one that iterates over the neighboring particles.

In addition to the baseline TinySTM, a straightforward ordered TinySTM version has been implemented, in which a transaction commits only when all the preceding ones have committed, stalling the thread until such a condition is fulfilled (or the transaction is aborted). The abort policy implies always aborting the subsequent transaction in case of conflict. This version will be referred to as TinySTM ordered and it will be also taken as a reference point, mainly in those cases where the order of computations is mandatory.

To mitigate random fluctuations in results due to the transactional behavior, each execution has been repeated at least 50 times, obtaining average measurements. Metrics measured from experiments include speed-up, taking the execution time of the sequential code version as the reference (but, if the sequential code is not available, the baseline TinySTM implementation with one thread is used instead); TCR (Transaction Commit Rate) [Ansari et al. 2008], as the percentage of committed transactions out of all executed ones, which measures the effectiveness of the parallelism exploitation; and throughput, as the number of committed transactions per second.

\section{STAMP}

STAMP benchmark codes have been configured using the workloads summarized in Table VI. The following experiments are focused on comparing TEPO versus both the original and ordered versions of TinySTM. TEPO was used inside loops with chunks of one iteration per transaction, as STAMP codes are already written transactionally, and they were executed as provided, without additional modifications.

For the purpose of testing conveniently both TEPO and TinySTM-ordered, an artificial transaction order has been forced for these two implementations according to the transaction starting time. However, base TinySTM was tested for unordered transactions. Note that the result of the execution of the STAMP codes is correct regardless of the order of transactions. This ordering introduces an execution-commit delay with respect to the baseline TinySTM (unordered commit) as explained in Figure 6. While the baseline TinySTM version can make transactions commit as soon as their execution ends, TinySTM-ordered cannot commit any transaction until the precedent 
Table VI. STAMP Workloads

\begin{tabular}{|c|c|c|c|c|c|c|}
\hline Code & $\begin{array}{c}\text { Input } \\
\text { arguments }\end{array}$ & \#TX & $\begin{array}{l}\text { \#TX Reads } \\
\text { (avg.) }\end{array}$ & $\begin{array}{l}\text { \#TX Writes } \\
\text { (avg.) }\end{array}$ & $\begin{array}{l}\text { Time in } \\
\text { TX }\end{array}$ & Contention \\
\hline Bayes & 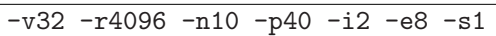 & $2 \mathrm{~K}$ & 28.75 & 3.26 & $99 \%$ & High \\
\hline Genome & -g16384 -s64 -n16777216 & $2489 \mathrm{~K}$ & 36.23 & 0.89 & $98 \%$ & Low \\
\hline Intruder & $-\mathrm{a} 10-1128-\mathrm{n} 65536-\mathrm{s} 1$ & $5816 \mathrm{~K}$ & 22.40 & 1.60 & $77 \%$ & High \\
\hline Kmeans & $\begin{array}{l}-\mathrm{m} 15-\mathrm{n} 15-\mathrm{t} 0.00001-\mathrm{i} \\
\quad \text { rand-n65536-d32-c16 }\end{array}$ & $4106 \mathrm{~K}$ & 599.99 & 599.99 & $1.5 \%$ & Low \\
\hline Labyrinth & -i random-x512-y512-z7-n512 & $1 \mathrm{~K}$ & 180.52 & 177.02 & $100 \%$ & High \\
\hline Ssca2 & $-\mathrm{s} 20-\mathrm{i} 1.0-\mathrm{u} 1.0-13-\mathrm{p} 3$ & $22362 \mathrm{~K}$ & 1.00 & 2.00 & $28 \%$ & Low \\
\hline Vacation & -n2 -q90 -u98 $-\mathrm{r} 1048576-\mathrm{t} 4194304$ & $4194 K$ & 284.01 & 5.54 & $97 \%$ & Low/Med. \\
\hline Yada & -a15 -i ttimeu1000000.2 & $2415 \mathrm{~K}$ & 58.74 & 18.01 & $96 \%$ & Medium \\
\hline
\end{tabular}
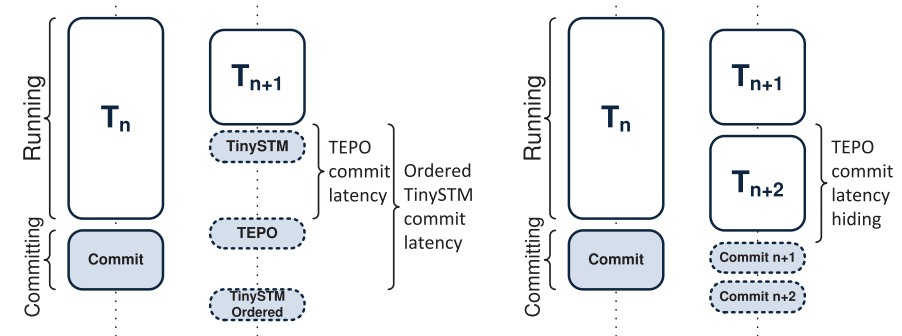

Fig. 6. Commit scenarios: in the baseline TinySTM, a transaction can commit just after its execution; TEPO commits when all precedent transactions have finished their execution (although not committed). In the straightforward ordered TinySTM version, a transaction commits when all precedent transactions are already committed. Additionally, TEPO can maintain several executed but not committed transactions, hiding the time waiting to commit.

transactions has been successfully committed. This situation leads to a commit delay that has an influence on performance. On the other hand, while preserving the precedent order, TEPO allows transactions to start the commit phase when the precedent transaction subset has been, at least, executed. This condition may reduce significantly the delay before committing, which has an effect on the benchmark execution time. Additionally, TEPO can hide this latency by allowing a thread to start a subsequent transaction if the commit condition is not fulfilled.

The observed speedup is shown in Figure 7. As expected, in almost all cases, TEPO speedup is kept between TinySTM and TinySTM-ordered. TEPO does not take advantage of the unordered execution as the baseline TinySTM does, but it is able to improve the execution time with respect to the straightforward ordered version (see Figure 6). The goal of these experiments is not to make TEPO compete against TinySTM in codes without ordering constraint, because TEPO obeys an artificial order that hurts the performance regarding the baseline TinySTM. The purpose is to show that even introducing a forced order, TEPO is able to perform better than a straightforward ordered implementation. Even so, for some particular experiments, TEPO does not underperform with respect to the baseline version.

In a first group of codes, the baseline version exhibits a relatively good scalability, which is mainly limited by the memory features of the target machine. This group includes Genome, Labyrinth, and Vacation. Although the TEPO speedup is lower in these cases, it shows a similar scalability than the baseline and always outperforms TinySTM-ordered. In a second group, composed of Intruder, Kmeans, and Yada, the scalability of the baseline version is also limited by the code features for a large number of threads. In these cases, TEPO follows the same trend as the baseline. The codes Ssca2 and Bayes do not fall into any of the two groups. In Ssca2, as a result of a high 


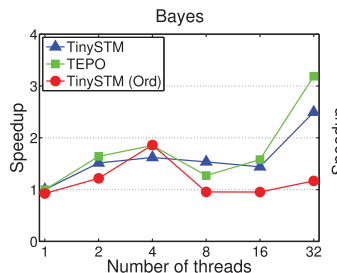

Intruder

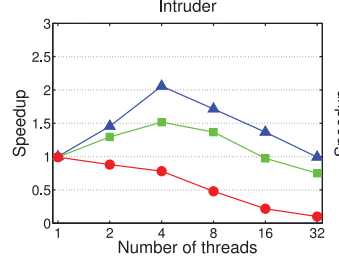

Labyrinth

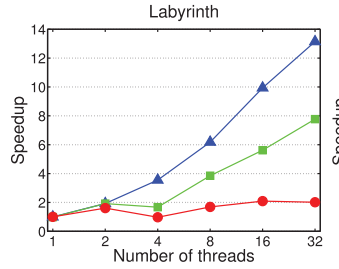

Vacation

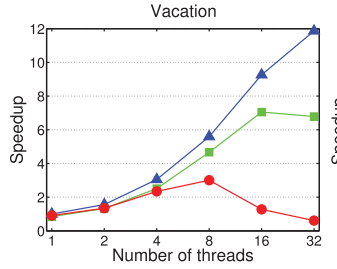

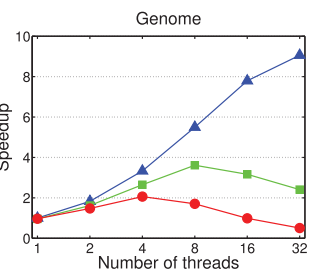

Kmeans

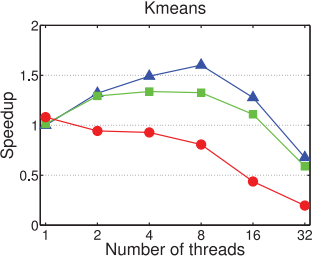

Ssca2

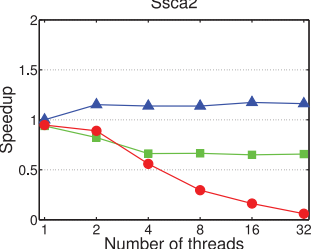

Yada

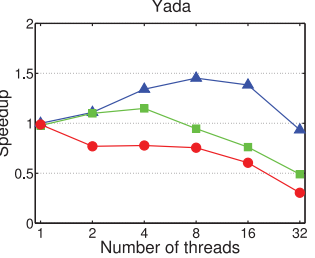

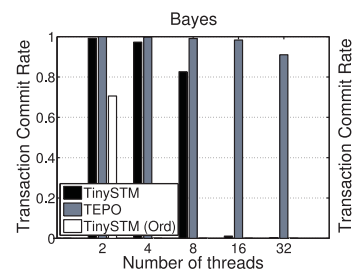

Intruder

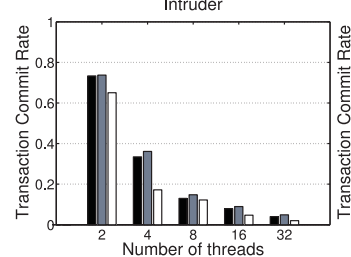

Labyrinth

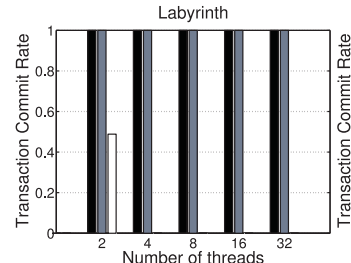

Vacation

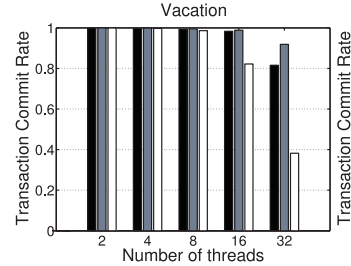

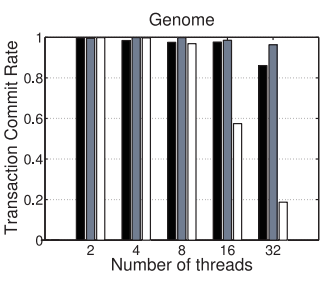

Kmeans

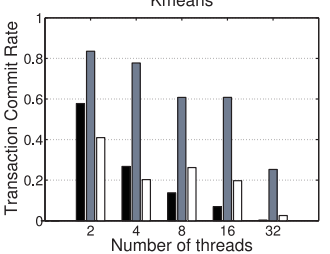

Ssca2

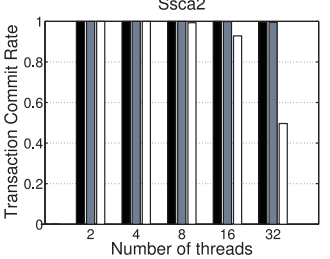

Yada

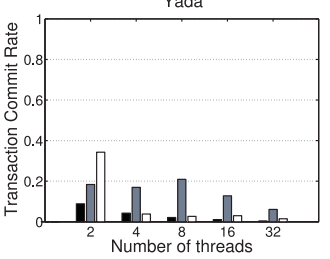

Fig. 7. STAMP benchmark: speedup and TCR using the configuration in Table VI.

number of very small transactions with low contention, all versions perform very poorly without extracting almost any parallelism. Nevertheless, TEPO continues to have an advantage over TinySTM-ordered. Finally, Bayes exhibits a mixed trend because the execution time is very sensitive to the order in which transactions are executed, which leads to variable behavior.

The commit delay associated with ordered implementations also has an effect in terms of TCR (Figure 7), as the commit delay can make the transaction lifetime larger, consequently increasing the probability of conflicts. The lower commit delay in TEPO allows a significant reduction of the abort rate regarding TinySTM-ordered. However, this is not the only important issue. Although in base TinySTM, transactions commit with no specific order, TEPO reaches better or similar TCR measures in most experiments. The artificial transaction order introduced by TEPO helps to filter out a high number of false data dependencies, which results in numerous transaction aborts for the baseline version. This effect is observed in the TCR scalability. TEPO TCR scales better than the TinySTM versions, especially for a high number of threads, as the contention is higher. However, this does not turn into a speedup improvement due to the negative effect of the artificial order.

TEPO TCR ranges from $70 \%$ to $100 \%$ for all codes except those exhibiting a limited speedup and lack of scalability (Intruder, Kmeans, and Yada). For these codes, TM systems are serializing the execution as a consequence of the high abort rate due to data conflicts. At one extreme, Yada exhibits the highest abort rate because of its large 


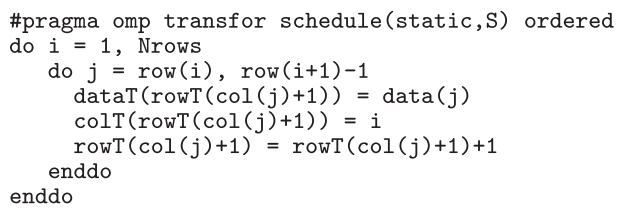

Fig. 8. Sparse matrix transposition main loop.

\begin{tabular}{cccc}
\hline Matrix & Size & $\begin{array}{c}\text { \#non-null coef. } \\
\text { per row (avg.) } \\
\text { (NNR) }\end{array}$ & $\begin{array}{c}\text { \#non-null coef. } \\
\text { per row / row size } \\
\text { (NNRD) }\end{array}$ \\
\hline beacx & $497 \times 506$ & 100 & $20.2 \%$ \\
wm3 & $207 \times 260$ & 14 & $6.7 \%$ \\
jpwh_991 & $991 \times 991$ & 6.1 & $0.62 \%$ \\
1138_bus & $1138 \times 1138$ & 3.6 & $0.32 \%$ \\
bcsstk18 & $11948 \times 11948$ & 12 & $0.10 \%$ \\
bcspwr10 & $5300 \times 5300$ & 4.1 & $0.07 \%$ \\
\hline
\end{tabular}

Fig. 9. Sparse matrix features.

transactions and the long time spent in transactions (around 96\%), which makes data conflicts more likely.

\section{Iterative Codes}

This second set of experiments includes two iterative irregular codes: the transposition of a sparse matrix and a code with histogram reductions. For the first code, as iterations cannot be rearranged, base TinySTM is not applicable. As a result, experiments compare only TEPO with TinySTM-ordered. However, the second code allows reordering the execution of the iterations, so all three TM implementations can be used.

The main loop of Pissanetzky's sparse matrix transposition is shown in Figure 8. Input and output matrices are given in well-formed CRS (compressed row storage), being defined by three unidimensional vectors: row, column, and data. The loop exhibits a complex memory access pattern with several indirection levels. Observe that there are three sources of dependencies. First, a loop-carried dependence is caused by the subscript rowT $(\operatorname{col}(j)+1)$ in the updating of vectors dataT and $\operatorname{colT}$, because the value of rowT can be calculated in a different iteration. A second loop-carried dependence is given by the reduction operation on rowT. Third, a possible output dependence is given by the writes in dataT and colT. However, this dependence is not relevant because in a well-formed CRS matrix, each position of dataT and colT is written only once in memory. Dependencies between iterations are determined by the fill-in of the input matrix, which is unknown before execution. Therefore, the behavior of the transactional system can be changed by using matrices with different features. A set of matrices taken from the Matrix Market repository [Boisvert et al. 1997], whose features are summarized in Figure 9, has been selected for evaluation.

The number of data conflicts, and consequently the effectiveness of the transactional parallelization, will be related to the density of nonnull rows in the input matrix, rather than the density of the whole matrix, as these rows are precisely those to be transposed. According to its average nonnull row density (NNRD), tested matrices can be classified into three classes: high NNRD (beacxc, wm3), medium NNRD (jpwh_991, 1138_bus), and low NNRD (bcsstk18, bcspwr10). The observed performance is depicted in Figure 10. In terms of throughput, the higher the NNRD is, the better performance that TEPO offers versus TinySTM-ordered. This relative trend is also followed by TCR measurements. Note that TCR is lower for smaller matrices, although TEPO outperforms TinySTM-ordered because just these matrices have the highest nonnull row density. Just because the outer loop has been parallelized, larger matrices will have more iterations in the inner loop, resulting in a higher absolute workload per outer loop iteration, and consequently, fewer transactions are executed per time unit. In all these experiments, TEPO was able to exploit more concurrency than the baseline ordered system.

Observe that the sparse matrix transposition is a strongly memory-bound code whose main operation is basically data moving. In order to analyze the scalability with the number of threads, the code has been evaluated adding an extra synthetic computational workload by operating over each matrix coefficient read in each iteration, before 


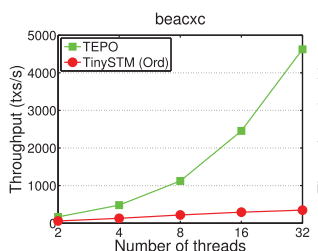

jpwh 991

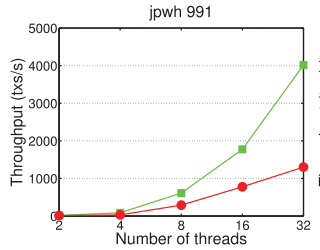

bcspwr10

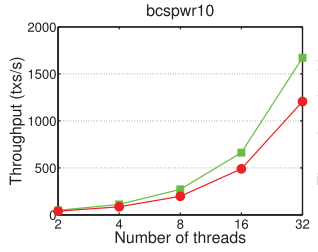

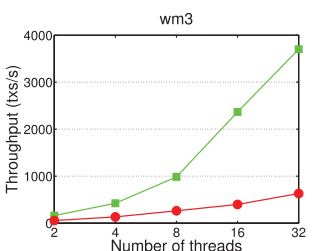

1138 bus

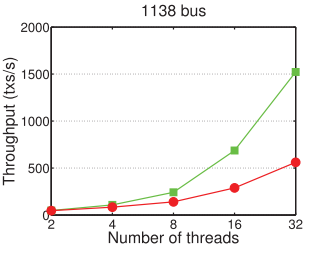

bcsstk18

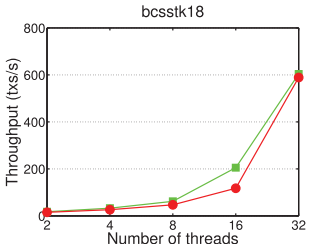

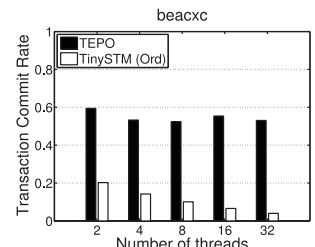

jpwh 991

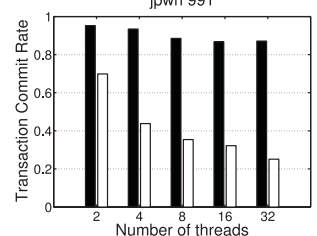

bespwr10

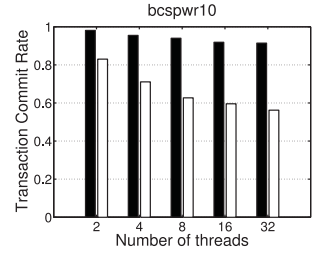

wm3

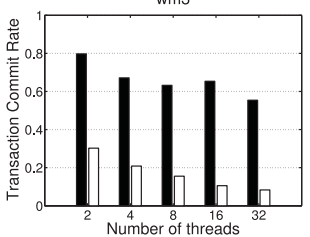

1138 bus

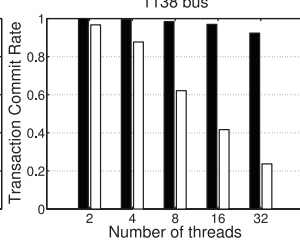

besstk 18

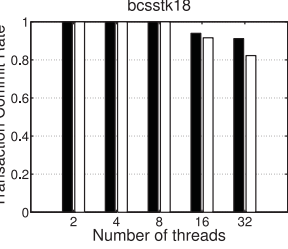

Fig. 10. Sparse matrix transposition: throughput and TCR for different input matrices using TEPO and TinySTM-ordered.
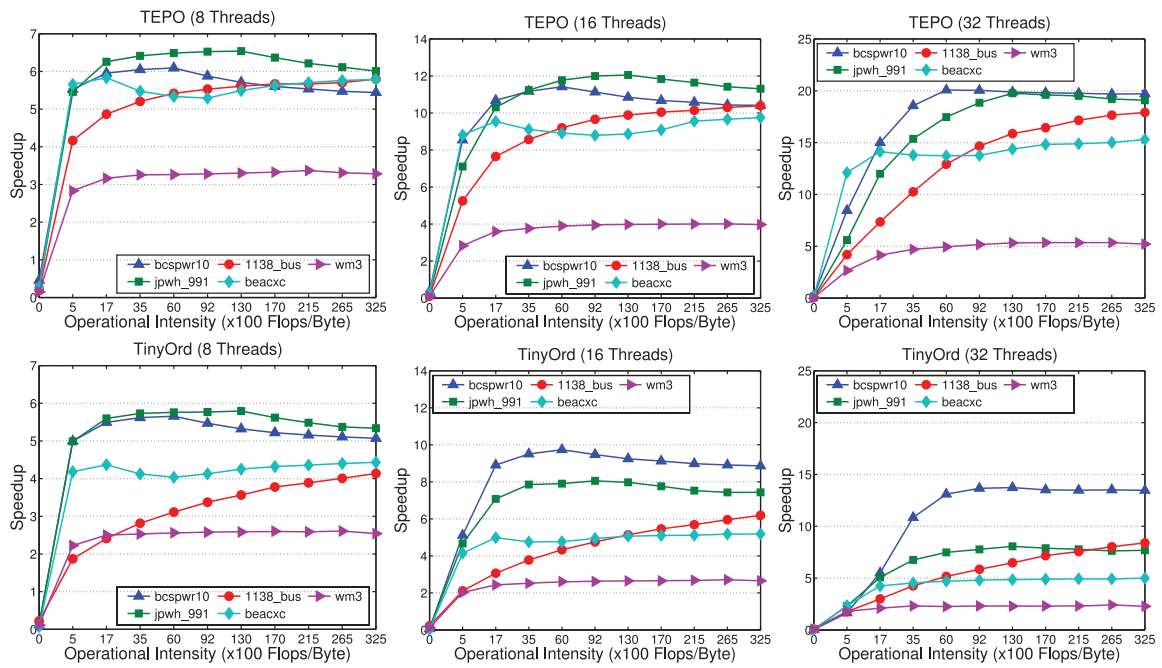

Fig. 11. Speedups for the sparse matrix transposition with extra workload $(8,16$, and 32 threads; $\mathrm{x}$-axis is on a quadratic scale).

being written in the transposed matrix. Results are shown in Figure 11, where speedup is summarized for several values of the synthetically added computational intensity [Williams et al. 2009]. As expected, the higher the operational intensity, the more exploitable the parallelism is in all cases, as the effect of the memory system vanishes. On the contrary, from the transactional point of view, higher operational intensity will involve larger transactions and consequently a higher abort cost. The advantage of TEPO over TinySTM-ordered is kept in almost all situations, especially for a large number of threads. Specifically, for eight threads, TEPO improves the speedup around 


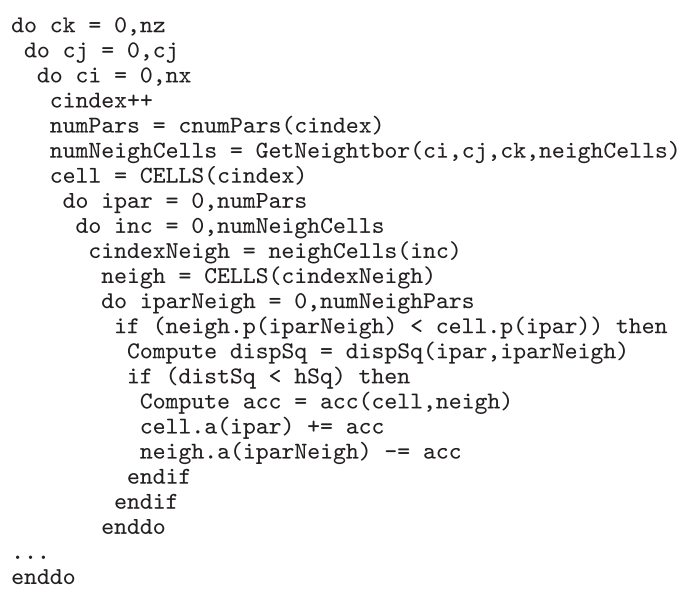

Fig. 12. Reduction loop in Fluidanimate.
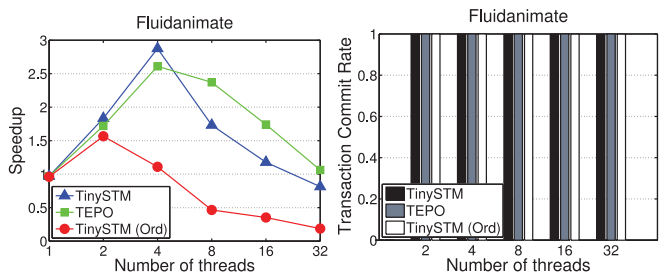

Fig. 13. Fluidanimate: TEPO vs. TinySTM and TinySTM-ordered.

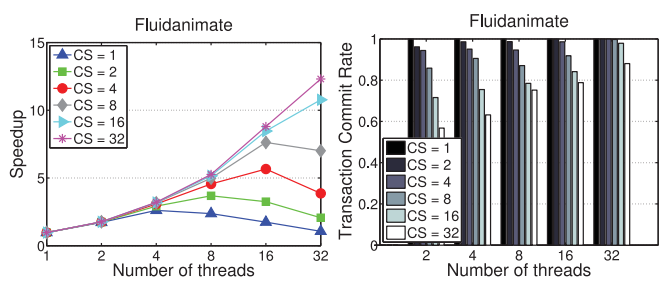

Fig. 14. Fluidanimate: TEPO speedup and TCR for several chunk sizes (CS) (iterations/chunk).

$10 \%$ in most cases, for 16 threads around $30 \%$, and for 32 threads around $60 \%$ for some matrices. TEPO results depend on the input matrix fill-in pattern, resulting in a convergent trend toward a maximum attainable parallelism. This trend is not exhibited by TinySTM-ordered, whose observed speedup falls below TEPO. Due to its small size, the speedup for the wm3 matrix is poor.

Fluidanimate, from the PARSEC benchmark suite [Bienia et al. 2008], is an iterative code solving a simplified version of the Navier-Stokes equations. It contains irregular histogram reduction loops [Gutiérrez et al. 2000], as sketched in Figure 12. About $96.1 \%$ of the sequential execution time is spent in them (functions ComputeForces and ComputeDensities). Evaluation is shown in Figures 13 and 14 (input set used: simlarge, $300 \mathrm{~K}$ particles, and five frames). Observe that the commutative and associative properties of reductions make TinySTM results correct, although iterations might have been disordered. In any case, TEPO obtains the best results of all methods. Note that for this code, only computation associated to boundary particles are enclosed in lock sections in the original source code, and consequently the conflict probability is low (only about $1.5 \%$ of writes in the reduction array involve potential conflicts). The chunk size has a significant influence on the scalability of TEPO, as the overhead/payload ratio is less important with large chunks. Nevertheless, the TCR worsens because the conflict probability increases (transactions with longer lifespan).

\section{Evaluating Overheads}

Software overhead associated with current STM implementations is usually not low. In order to evaluate the overhead of our proposal with respect to the baseline system, two 2D stencil benchmark codes from Polybench [Pouchet 2011] have been used. Note that these are memory-bound codes [Christen et al. 2011], with low operational intensity. The first code, Jacobi, can run fully in parallel because computation and memory update stages are decoupled by means of an auxiliary matrix (see Figure 15(a)). On the other hand, Seidel code computes in place, and hence, its execution is serialized due to the loop-carried dependencies (see Figure 15(b)). 


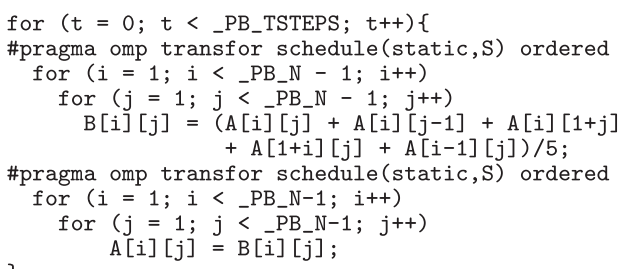

(a)

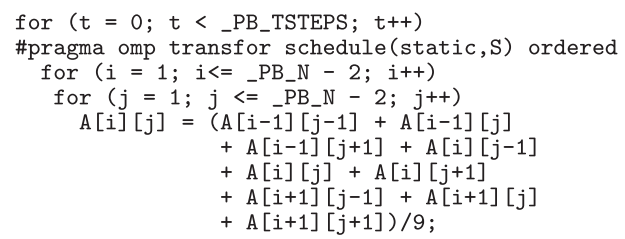

Fig. 15. Computational kernel of stencil codes from Polybench: (a) Jacobi 2D, (b) Seidel 2D.
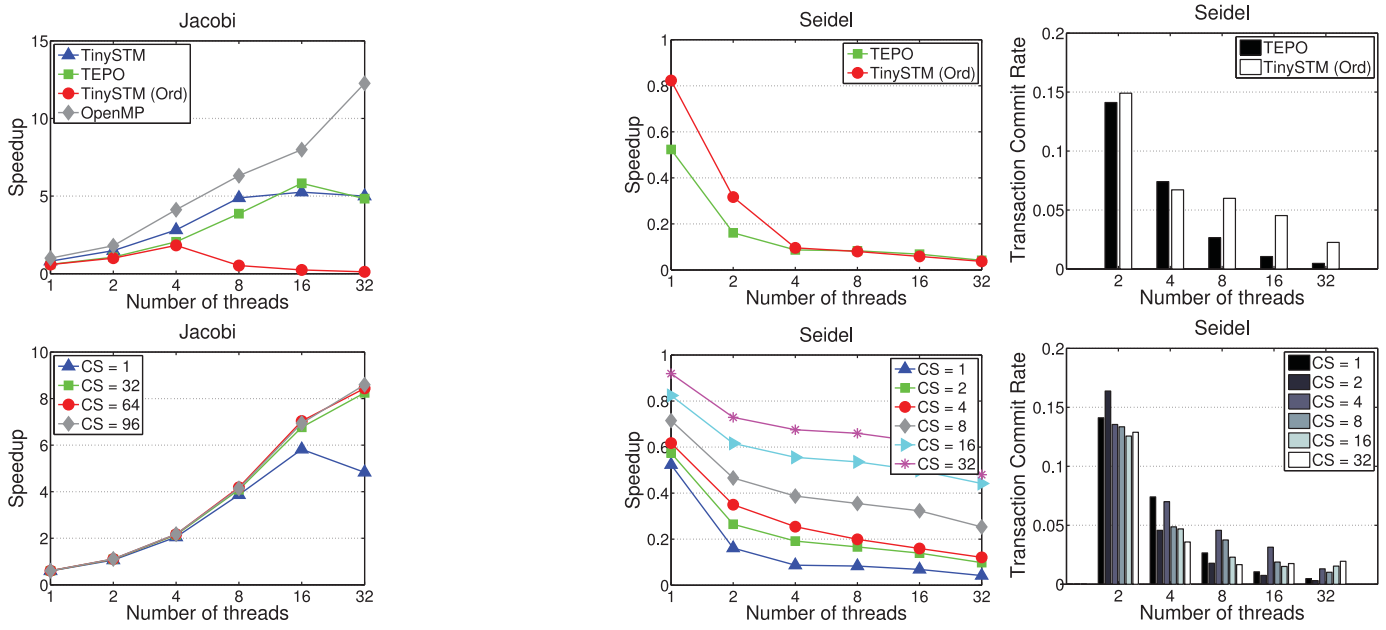

Seidel
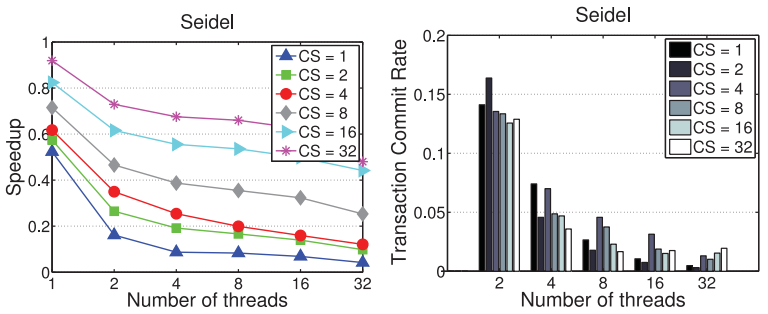

Fig. 17. Seidel 2D speedup and TCR.

Speedup measurements for Jacobi are shown in Figure 16. On being fully parallel, TCR has been omitted (no transactional conflicts). As a reference, a simple parallelization using OpenMP is also shown (each loop parallelized separately with the pragma omp parallel for clause). Since reordering parallel iterations does not affect the output results, the baseline TinySTM is applicable. The main point here is that the TEPO parallel version does not add a significant extra overhead over the baseline TM system. Even more, a slight advantage of TEPO is observed as the number of threads grows. By increasing the chunk size, the overhead can be better hidden with concurrency, improving speedup to around $75 \%$ of that of OpenMP.

The sequential nature of Seidel involves a strong worsening in the speedup and TCR as displayed in Figure 17. As iterations cannot be reordered, the TinySTM-ordered has been used as a reference. The low computation workload per iteration and the overhead of the STM system are responsible for the efficiency downfall, much below the sequential version, which becomes worse for a high number of threads. Even so, TEPO does not perform worse than TinySTM-ordered. As before, this poor behavior can be alleviated by increasing the chunk size. As Seidel is in place, consecutive transactions have conflicts. Therefore, as TEPO allows a higher number of active transactions than TinySTM-ordered, the abort rate is larger. This fact explains the lower TCR for TEPO, as TinySTM-ordered only needs to abort one transaction (the current one) while TEPO may abort a chain of transactions per thread. 


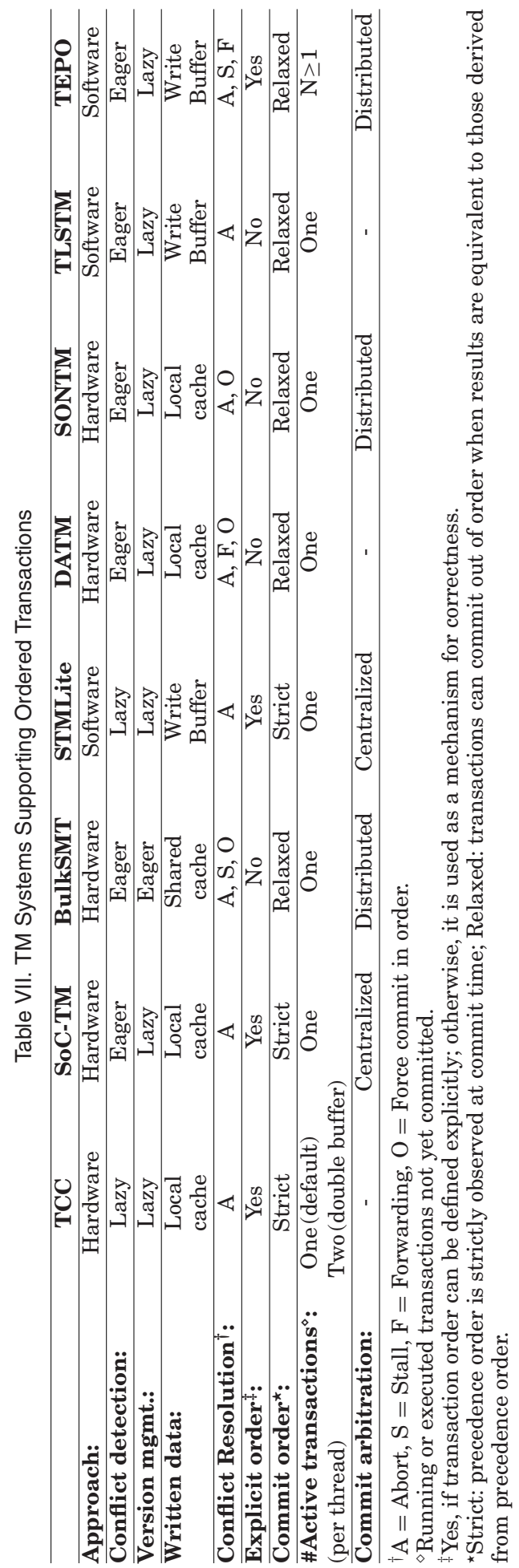




\section{RELATED WORK}

Two major approaches to optimistic parallelism are TM and TLS. TM focuses on optimistic thread synchronization of a multithreaded program, and TLS focuses on the speculative parallelization of a sequential program. A main difference between TM and TLS is that the latter assumes that the sequential semantics of the original program are maintained in the parallel execution, while TM is unordered. However, both concepts are strongly related, as stated in Porter et al. [2009], where the problem of using TM to support speculative multithreading is analyzed. In a similar direction, the proposed TEPO model leverages TM to support speculative execution of transactions subjected to a user-defined precedence order that can represent the essential sequential semantics of the program.

Despite the original TM concept not considering ordering between transactions, order can be a strong tool to improve some aspects related to the execution of transactions. Some TM systems make use of order as a mechanism to solve data conflicts by reordering transaction commits. This is the case for systems such as BulkSMT [Qian et al. 2012], DATM [Ramadan et al. 2008], and SONTM [Aydonat and Abdelrahman 2010]. In these systems, the commit precedence order comes from the eventual data conflicts. In TEPO, on the contrary, the precedence order is defined by the user and it determines how the data conflicts will be managed. On the other hand, other TM proposals, such as SoC-TM [Ferri et al. 2011], STMLite [Mehrara et al. 2009], and TCC [Hammond et al. 2004], obey some established precedence order. SoC-TM and STMLite define the precedence order according to the sequential semantics of the original program. Both systems rely on a centralized commit arbitrator to guarantee the order, while TEPO carries out this process in a fully distributed way. In TCC, the precedence order is defined by means of phase numbers that are assigned to the transactions by the user. Transactions with the same number are executed unordered, while a barrier is introduced between transactions with consecutive phase numbers. This technique does not allow one to define a partial precedence order, unlike TEPO. A remarkable property of TEPO is that a single thread can maintain several active transactions (thanks to decoupling execution from commit), in contrast to the previous proposals that support only one per thread (except TCC, which may support two transactions using double buffering). TLSTM [Barreto et al. 2012] combines both TM and TLS approaches. All transactions execute unordered, but each one is split into several smaller tasks that are speculatively executed in parallel (ordered).

Table VII summarizes some of the most relevant properties of the discussed ordered TM systems, in comparison to TEPO.

\section{CONCLUSIONS}

We have presented the TEPO model aimed at improving concurrency in parallel transactional codes that are subjected to some precedence order restrictions required for correctness. In addition, as the precedence order is used to filter out data conflicts, the model offers the opportunity to reduce the impact of conflicts in general codes by introducing an explicit user-defined order. The support for precedence order is combined with the possibility of handling several transactions in a single thread.

A lightweight STM system (TinySTM) has been extended to support the TEPO model and has been evaluated in an ample set of benchmarks. Results show that in iterative codes with order restrictions, our system improves concurrency significantly, while in codes with no order restrictions, TEPO is able to outperform the base TM system for most tested codes. On the other hand, experiments show that TEPO does not introduce major overheads to the base TM system. 


\section{REFERENCES}

M. Ansari, C. Kotselidis, K. Jarvis, M. Luján, C. Kirkham, and I. Watson. 2008. Advanced Concurrency Control for Transactional Memory Using Transaction Commit Rate. In 14th Int'l. Conf. on Parallel Processing (Euro-Par'08). 719-728.

Utku Aydonat and Tarek S. Abdelrahman. 2010. Hardware Support for Relaxed Concurrency Control in Transactional Memory. In 43rd Ann. IEEE/ACM Int'l. Symp. on Microarchitecture (MICRO'10). 15-26.

W. Baek, C. C. Minh, M. Trautmann, C. Kozyrakis, and K. Olukotun. 2007. The OpenTM Transactional Application Programming Interface. In 16th Int'l. Conf. on Parallel Architectures and Compilation Techniques (PACT'07). 376-387.

Joao Barreto, Aleksandar Dragojevic, Paulo Ferreira, Ricardo Filipe, and Rachid Guerraoui. 2012. Unifying Thread-Level Speculation and Transactional Memory. In ACM/IFIP/USENIX 13th Int'l. Middleware Conf. 187-207.

Christian Bienia, Sanjeev Kumar, Jaswinder Pal Singh, and Kai Li. 2008. The PARSEC Benchmark Suite: Characterization and Architectural Implications. In 17th Int'l. Conf. on Parallel Architectures and Compilation Techniques (PACT'08). 72-81.

Barna L. Bihari. 2013. Transaction Memory for Unstructured Mesh Simulations. J. Scientific Computing 54, 2-3 (2013), 311-332.

Ronald F. Boisvert, Roldan Pozo, Karin Remington, Richard F. Barrett, and Jack J. Dongarra. 1997. Matrix Market: A Web Resource for Test Matrix Collections. In IFIP TC2/WG2.5 Working Conf. on Quality of Numerical Software: Assessment and Enhancement. 125-137.

Chi Cao Minh, JaeWoong Chung, Christos Kozyrakis, and Kunle Olukotun. 2008. STAMP: Stanford Transactional Applications for Multi-Processing. In IEEE Int'l. Symp. on Workload Characterization (IISWC'08).

Matthias Christen, Olaf Schenk, and Helmar Burkhart. 2011. Automatic Code Generation and Tuning for Stencil Kernels on Modern Shared Memory Architectures. J. Computer Science - Research and Development 26, 3-4 (2011), 205-210.

Matthew DeVuyst, Dean M. Tullsen, and Seon Wook Kim. 2011. Runtime Parallelization of Legacy Code on a Transactional Memory System. In 6th Int'l. Conf. on High Performance and Embedded Architectures and Compilers (HiPEAC'11).

Pascal Felber, Christof Fetzer, and Torvald Riegel. 2008. Dynamic Performance Tuning of Word-Based Software Transactional Memory. In 13th ACM SIGPLAN Symp. on Principles and Practice of Parallel Programming (PPoPP'08).

C. Ferri, A. Marongiu, B. Lipton, R. Bahar, T. Moreshet, L. Benini, and M. Herlihy. 2011. SoC-TM: Integrated HW/SW Support for Transactional Memory Programming on Embedded MPSoCs. In IEEE/ACM/IFIP Int'l. Conf. on Hardware / Software Codesign and System Synthesis (CODES+ISSS'11). 39-48.

D. Geer. 2005. Industry Trends: Chip Makers Turn to Multicore Processors. IEEE Computer 38, 5 (2005), 11-13.

M. Angel Gonzalez-Mesa, Ricardo Quislant, Eladio Gutierrez, and Oscar Plata. 2012. Automatic Loop Parallelization Using Transactional Memory Support. In 16th Workshop on Compilers for Parallel Computers (CPC'12).

Allan Gottlieb, Boris D. Lubachevsky, and Larry Rudolph. 1983. Basic Techniques for the Efficient Coordination of Very Large Numbers of Cooperating Sequential Processors. ACM Trans. on Programming Languages and Systems (TOPLAS) 5, 2 (April 1983), 164-189.

E. Gutiérrez, O. Plata, and E. L. Zapata. 2000. A Compiler Method for the Parallel Execution of Irregular Reductions in Scalable Shared Memory Multiprocessors. In 14th Int'l. Conf. on Supercomputing (ICS'O0). 78-87.

Lance Hammond, Vicky Wong, Mike Chen, Brian D. Carlstrom, John D. Davis, Ben Hertzberg, Manohar K. Prabhu, Honggo Wijaya, Christos Kozyrakis, and Kunle Olukotun. 2004. Transactional Memory Coherence and Consistency. In 31st Ann. Int'l. Symp. on Computer Architecture (ISCA'04). 102-113.

Tim Harris, James R. Larus, and Ravi Rajwar. 2010. Transactional Memory (2nd Ed.). Morgan \& Claypool.

Maurice Herlihy. 1991. Wait-Free Synchronization. ACM Trans. on Programming Languages and Systems (TOPLAS) 13, 1 (January 1991), 124-149.

Maurice Herlihy and J. Eliot B. Moss. 1993. Transactional Memory: Architectural Support for Lock-Free Data Structures. In 20th Ann. ACM / IEEE Int'l. Symp. on Computer Architecture (ISCA'93). 289-300.

Mojtaba Mehrara, Jeff Hao, Po-Chun Hsu, and Scott Mahlke. 2009. Parallelizing Sequential Applications on Commodity Hardware Using a Low-Cost Software Transactional Memory. In ACM SIGPLAN Conf. on Programming Language Design and Implementation (PLDI'09). 166-176.

Samuel P. Midkiff. 2012. Automatic Parallelization: An Overview of Fundamental Compiler Techniques. Morgan \& Claypool. 
S. Pissanetzky. 1984. Sparse Matrix Technology. Academic Press.

Leo Porter, Bumyong Choi, and Dean M. Tullsen. 2009. Mapping Out a Path from Hardware Transactional Memory to Speculative Multithreading. In 18th Int'l. Conf. on Parallel Architectures and Compilation Techniques (PACT'09).

Louis-Noel Pouchet. 2011. PolyBench: The Polyhedral Benchmark Suite. Available at http://www.cse.ohiostate.edu/pouchet/software/polybench/.

Xuehai Qian, Benjamin Sahelices, and Josep Torrellas. 2012. BulkSMT: Designing SMT Processors for Atomic-Block Execution. In 18th IEEE Int'l. Symp. on High Performance Computer Architecture (HPCA'12). 1-12.

Hany E. Ramadan, Christopher J. Rossbach, and Emmett Witchel. 2008. Dependence-Aware Transactional Memory for Increased Concurrency. In 41st Ann. IEEE/ACM Int'l. Symp. on Microarchitecture (MICRO'08). 246-257.

Torvald Riegel, Christof Fetzer, and Pascal Felber. 2007. Time-Based Transactional Memory with Scalable Time Bases. In 19th Annual ACM Symp. on Parallel Algorithms and Architectures (SPAA'07). 221-228.

Mohamed M. Saad, Mohamed Mohamedin, and Binoy Ravindran. 2012. HydraVM: Extracting Parallelism from Legacy Sequential Code Using STM. In 4th USENIX Conf. on Hot Topics in Parallelism (HotPar'12).

Martin Schindewolf, Barna Bihari, John Gyllenhaal, Martin Schulz, Amy Wang, and Wolfgang Karl. 2012. What Scientific Applications Can Benefit from Hardware Transactional Memory? In Int'l. Conf. on High Performance Computing, Networking, Storage and Analysis (SC'12).

Gurindar S. Sohi, Scott E. Breach, and T. N. Vijaykumar. 1995. Multiscalar Processors. In 22nd Ann. ACM / IEEE Int'l. Symp. on Computer Architecture (ISCA'95). 414-425.

Samuel Williams, Andrew Waterman, and David Patterson. 2009. Roofline: An Insightful Visual Performance Model for Multicore Architectures. Commun. ACM 52, 4 (2009), 65-76.

Richard M. Yoo, Christopher J. Hughes, Konrad Lai, and Ravi Rajwar. 2013. Performance Evaluation of Intel Transactional Synchronization Extensions for High-Performance Computing. In Int'l. Conf. on High Performance Computing, Networking, Storage and Analysis (SC'13).

Received October 2013; revised February 2014; accepted April 2014 